\title{
An Institution-Independent Generalization of Tarski's Elementary Chain Theorem
}

\author{
Daniel Găină and Andrei Popescu \\ Şcoala Normală Superioară, Bucharest ${ }^{\star \star}$ \\ gaina_daniel@yahoo.com, uuomul@yahoo.com
}

\begin{abstract}
We prove an institutional version of Tarski's Elementary Chain Theorem applicable to a whole plethora of "first-order-accessible" logics, which are, roughly speaking, logics whose sentences can be constructed from atomic formulae by means of classical first-order connectives and quantifiers. These include the unconditional equational, positive, $(\Pi \cup \Sigma)_{n}^{0}$, and full first-order logics, as well as less conventional logics, used in computer science, such as hidden or rewriting logic.
\end{abstract}

Keywords: institution, elementary morphism, elementary chain property.

\section{Introduction}

The notion of elementary embedding is an important one in classical first-order model theory [4]. Elementary chains (i.e., chains of elementary embeddings) are known to be a fundamental proof tool for results regarding preservation of satisfaction, axiomatizability, Robinson consistency, Craig interpolation [4], saturated models, stability, categoricity in power [30], and many others. The extension of elementary embeddings to infinitary logics $[18,21,17]$ reveals the need in mathematical logic for accommodating this notion in other logical systems too. And the monograph [18] actually shows that this is a natural and very fruitful thing to do.

The present paper introduces and studies abstract notions of elementary embedding and elementary chain, in the framework of institutions [14], and points out many particular cases. Two aspects motivate and justify our study:

- the mentioned importance of elementary embeddings in model theory;

- the logic-independent status of our concepts and results.

Besides its intrinsic abstract model-theoretic contribution, our study might be of interest for the theory of formal specifications, where a logic-independent view is desirable for as long as possible in the specification process, and where structural

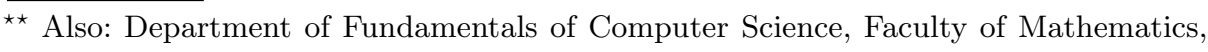
University of Bucharest. 
properties usually approachable by means of elementary chains, such as Craig interpolation or axiomatizability, are crucial.

Institutions are abstract logical frameworks which provide a category of signatures (languages) and signature morphisms (language translations), and, for each signature, a set of sentences, a category of models, and a satisfaction relation. Sentences have translations, and models have reducts, along signature morphisms; the translations and reducts express the sentence- and model- modifications under change of notation from one language to another. Satisfaction is required to be invariant under change of notation. More abstract than the general logics of [1], institutions were introduced as frameworks for building model theory for computer science, in a logic-independent way. Thus general institutional results were applicable to the diversity of logical systems used in computer science. Besides their great generality, another important feature of institutions, not present, or poorly present, in other abstract frameworks, is the flexible support for language translations. This feature, particularly useful in formal specification and the semantics of programming languages, is also interesting from a logical point of view. As shown in $[34,35,7,10,8,6]$ and other places, signature morphisms (language translations) turn out to be a very insightful tool for finding concrete structure in the core of abstract logic. For example, any institution hides inside a "first-order logic", which can be uncovered by means of basic sentences, logical connectives, and quantifications over some signature morphisms (see Sections 2 and 3). ${ }^{1}$

Our institutional notion of elementary embedding (that we call "elementary morphism") also uses signature morphisms in an essential way, by defining elementarity as preservation of satisfaction in expansions along certain signature morphisms. Recall that, classically, an elementary embedding between two models $A$ and $B$ of the same language is a model-embedding $A \stackrel{h}{\rightarrow} B$ such that for each formula $e\left(x_{1}, \ldots, x_{n}\right)$ and each sequence $a_{1}, \ldots, a_{n} \in A, A \models e\left(a_{1}, \ldots, a_{n}\right)$ iff $B \models e\left(h\left(a_{1}\right), \ldots, h\left(a_{n}\right)\right)$. Because of the existence of negations, the "iff" above can be replaced by "implies". In order to abstract away this concept, we follow an idea originating in [34] which treats (non-closed) $\Sigma$-formulae as sentences in signature "extensions" $\varphi: \Sigma \rightarrow \Sigma^{\prime}$. And we use quasi-representable signature morphisms [6] to capture the requirement that the "extension" only adds "constants". ( $\varphi$ is quasi-representable if, for each $A^{\prime} \in\left|\operatorname{Mod}\left(\Sigma^{\prime}\right)\right|$, the canonical functor $A^{\prime} / \operatorname{Mod}(\varphi): A^{\prime} / \operatorname{Mod}\left(\Sigma^{\prime}\right) \rightarrow A^{\prime} 1_{\varphi} / \operatorname{Mod}(\Sigma)$ is an isomorphism of categories; hence giving a $\Sigma^{\prime}$-morphism of source $A^{\prime}$ is equivalent to giving a $\Sigma$-morphism of source $A^{\prime} 1_{\varphi}$. This situation has the following intuitive explanation: all the "extra items" of $\Sigma^{\prime}$ w.r.t. $\Sigma$ being constant symbols, a $\Sigma$-morphism $A \stackrel{h}{\rightarrow} B$ can have only one $\Sigma^{\prime}$-expansion of given source $A^{\prime}$.) Quasi-representability of signature morphisms is a weakening of the concept of representability introduced in [7]. By fixing a class $\mathcal{Q}$ of quasi-representable signature morphisms, we call a $\Sigma$-morphism $A \stackrel{h}{\rightarrow} B \mathcal{Q}$-elementary if for each signature morphism $\varphi: \Sigma \rightarrow \Sigma^{\prime}$

\footnotetext{
${ }^{1}$ Compare this natural appearance of a first-order sublogic with the need to explicitly
} postulate the existence of such a sublogic in the context of general logics of [1]. 
in $\mathcal{Q}$, each $\varphi$-expansion $A^{\prime} \stackrel{h^{\prime}}{\rightarrow} B^{\prime}$ of $h$, and each $\Sigma$-sentence $e^{\prime}, A^{\prime} \models e^{\prime}$ implies $B^{\prime} \models e^{\prime}$. (See Section 4 for a detailed motivation of this definition.)

One can alternatively define elementary morphisms by elementary diagrams. Classically, the elementary diagram [4] $E D g(A)$ of a model $A$ is the set of all sentences in $\Sigma(A)$ (the language $\Sigma$ of $A$ extended with all elements of $A$ as constants), true in $A$. Then a model-inclusion $A \stackrel{h}{\rightarrow} B$ is elementary iff $E D g(A) \subseteq$ $E D g(B)$. Thanks to a recent concept of institutional diagram [8], we can also define institutionally elementary morphisms by diagrams (we abbreviate these as $d$-elementary morphisms). The diagrams of [8] provide, for each signature $\Sigma$ and $\Sigma$-model $A$, a parameterized signature extension $\iota_{\Sigma}(A): \Sigma \rightarrow \Sigma_{A}$ and a selfparameterized $\iota_{\Sigma}(A)$-expansion of $A$. In addition, the diagrams are "functorial", i.e., they have corresponding structure for signature- and model- morphisms; in particular, any $\Sigma$-morphism $A \stackrel{h}{\rightarrow} B$ yields a signature morphism $\iota_{\Sigma}(h)$ : $\Sigma_{A} \rightarrow \Sigma_{B}$ such that $\iota_{\Sigma}(A) ; \iota_{\Sigma}(h)=\iota_{\Sigma}(B)$. We call $h$ d-elementary if, for all $\Sigma_{A}$-sentences $e^{\prime}, A_{A} \models e^{\prime}$ implies $B_{B} \models \iota_{\Sigma}(h)\left(e^{\prime}\right)$. d-elementarity is expressible more compactly than $\mathcal{Q}$-elementarity, but requires an amount of rather evolved extra structure on institutions.

Here is the structure of this paper. Section 2 recalls some notions regarding categories, institutions, and diagrams. Section 3 discusses and exemplifies the concepts, central in this paper, of (finitely) representable and (finitely) quasirepresentable signature morphism. Section 4 introduces elementary (model-) morphisms w.r.t. a class of quasi-representable signature morphisms. Section 5 proves an institutional version of Tarski's Elementary Chain Theorem, in the following slightly stronger form: elementary morphisms are closed under directed colimits. Section 6 introduces an alternative, diagrammatic version of elementary morphism and shows its equivalence to the previous notion under certain mild conditions (diagrams being in the considered class of quasi-representable morphisms and another condition that we call normality). Some concluding remarks end the paper.

\section{Preliminaries}

\section{Categories}

We assume that the reader is familiar with basic categorical notions like functor, natural transformation, colimit, comma category, etc. A standard textbook on the topic is [19]. We are going to use the terminology from there, with a few exceptions that we point out below. We use both the terms "morphism" and "arrow" to refer morphisms of a category. Composition of morphisms and functors is denoted using the symbol ";" and is considered in diagrammatic order.

Let $\mathcal{C}$ and $\mathcal{C}^{\prime}$ be two categories. Given an object $A \in|\mathcal{C}|$, the comma category of objects in $\mathcal{C}$ under $A$ is denoted $A / \mathcal{C}$. Recall that the objects of this category are pairs $(h, B)$, where $B \in|\mathcal{C}|$ and $A \stackrel{h}{\longrightarrow} B$ is a morphism in $\mathcal{C}$. Throughout the paper, we might let either $(A \stackrel{h}{\longrightarrow} B, B)$ or $(h, B)$ indicate objects in $A / \mathcal{C}$. 
A morphism in $A / \mathcal{C}$ between two objects $(h, B)$ and $(g, D)$ is just a morphism $B \stackrel{f}{\longrightarrow} D$ in $\mathcal{C}$ such that $h ; f=g$ in $\mathcal{C}$. There exists a canonical forgetful functor between $A / \mathcal{C}$ and $\mathcal{C}$, mapping each $(h, B)$ to $B$ and each $f:(h, B) \rightarrow(g, D)$ to $f: B \rightarrow D$. Also, if $F: \mathcal{C}^{\prime} \rightarrow \mathcal{C}$ is a functor, $A \in|\mathcal{C}|, A^{\prime} \in\left|\mathcal{C}^{\prime}\right|$, and $A \stackrel{u}{\rightarrow} F\left(A^{\prime}\right)$ is in $\mathcal{C}$, then there exists a canonical functor $u / F: A^{\prime} / \mathcal{C}^{\prime} \rightarrow A / \mathcal{C}$ mapping each $\left(A^{\prime} \stackrel{h}{\rightarrow} B, B\right)$ to $(u ; F(h), F(B))$ and each $f:(h, B) \rightarrow(g, D)$ to $F(f):(u ; F(h), F(B)) \rightarrow(u ; F(g), F(D))$. If $\mathcal{C}=\mathcal{C}^{\prime}$ and $F$ is the identity functor $1_{\mathcal{C}}$, we write $u / \mathcal{C}$ instead of $u / F$; and if $F\left(A^{\prime}\right)=A$ and $u=1_{A}$, we write $A^{\prime} / F$ instead of $u / F$.

Let $\mathcal{C}$ and $\mathcal{S}$ be two categories such that $\mathcal{S}$ is small. A functor $D: \mathcal{S} \rightarrow \mathcal{C}$ is also called a diagram. We usually identify a diagram $D: \mathcal{S} \rightarrow \mathcal{C}$ with its image in $\mathcal{C}, D(\mathcal{S})$. A cocone of $D$ is a natural transformation $\mu: D \Longrightarrow V$ between the functor $D$ and [the constant functor mapping all objects to $V$ and all morphisms to $\left.1_{V}\right] ; V$ is an object in $\mathcal{C}$, the vertex of the colimit, and the components of $\mu$ are the structural morphisms of the colimit. Any partially ordered set $(I, \leq)$ can be regarded as a category in the obvious way, with the arrows being pairs $i \leq j$. A non-empty partially ordered set $(I, \leq)$ is said to be directed if for all $i, j \in I$, there exists $k \in I$ such that $i \leq k$ and $j \leq k$, and is called a chain if the order $\leq$ is total. A diagram defined on a directed set (on a chain) shall be called directed diagram (chain diagram), and a colimit of such a diagram directed colimit (chain colimit). A final subset of a directed partially ordered set $(I, \leq)$ is a subset $K$ of $I$ such that for all $i \in I$, there exists $k \in K$ such that $i \leq k$. For instance, given $i \in I, I_{i}=\{j \in I \mid i \leq j\}$ is a final subset of $(I, \leq)$. A sub-diagram of a directed diagram $D:(I, \leq) \rightarrow \mathcal{C}$ is the restriction of $D$ to $(K, \leq)$, where $K$ is a subset of $(I, \leq)$; the sub-diagram is said to be final if $K$ is final. An object $A$ in a category $\mathcal{C}$ is called finitely presented if for each directed diagram $D:(I, \leq) \rightarrow \mathcal{C}$ with colimit $\left\{D i \stackrel{\mu_{i}}{\rightarrow} B\right\}_{i \in I}$, and for each morphism $A \stackrel{h}{\rightarrow} B$, there exists $j \in I$ and $A \stackrel{g}{\rightarrow} D j$ such that $g ; \mu_{j}=h$.

Let $\mathcal{C}^{\prime}$ be a subcategory of $\mathcal{C} . \mathcal{C}^{\prime}$ is called a broad subcategory if it contains all the objects of $\mathcal{C} . \mathcal{C}^{\prime}$ is said to be closed under directed colimits (chain colimits) if for any directed diagram (chain diagram) $D:(I, \leq) \rightarrow \mathcal{C}$ such that [for each $i \leq j$ in $I, D(i \leq j)$ is in $\left.\mathcal{C}^{\prime}\right]$, any colimit $\left\{D i \stackrel{\mu_{i}}{\rightarrow} B\right\}_{i \in I}$ of $D$ has all the structural morphisms $\mu_{i}$ in $\mathcal{C}^{\prime} . \mathcal{C}^{\prime}$ is said to be closed under pushouts if for each pushout $\left(A_{2} \stackrel{h_{2}}{\longleftarrow} A^{h_{1}} \rightarrow A_{1}, A_{2} \stackrel{h_{1}^{\prime}}{\rightarrow} A^{\prime h_{2}^{\prime}} \stackrel{A_{1}}{\stackrel{1}{\prime}}\right.$ in $\mathcal{C}, h_{1}^{\prime}$ is in $\mathcal{C}^{\prime}$ whenever $h_{1}$ is in $\mathcal{C}^{\prime}$. Note that the notion of "closed under" that we adopt is stronger for pushouts than for directed or chain colimits. The following lemma is proved in [19].

Lemma 1 Let $(I, \leq)$ be a directed set, $\mathcal{C}$ a category, $D:(I, \leq) \rightarrow \mathcal{C}$ a diagram, and $\left\{D i \stackrel{\mu_{i}}{\rightarrow} A\right\}_{i \in I}$ its colimit. If $K$ is a final subset of $(I, \leq)$, then $\left\{D i \stackrel{\mu_{i}}{\rightarrow} A\right\}_{i \in K}$ is a colimit of the corresponding final sub-diagram of $D$.

\section{Institutions}

Institutions were introduced in [14] with the original goal of providing an abstract, logic-independent framework for algebraic specifications of computer sci- 
ence systems. By isolating the essence of a logical system in the abstract satisfaction relation, these structures achieve an appropriate level of generality for the development of abstract model theory, as shown by a whole series of (old and new) papers: [34-36, 31, 32, 7, 8, 10, 9, 13, 27]. See also [26] for an up-to-date discussion on institutions as abstract logics.

An institution [14] consists of:

1. a category Sign, whose objects are called signatures.

2. a functor Sen $:$ Sign $\rightarrow$ Set, providing for each signature a set whose elements are called $(\Sigma$-) sentences.

3. a functor $\operatorname{Mod}:$ Sign $\rightarrow C a t^{o p}$, providing for each signature $\Sigma$ a category whose objects are called $(\Sigma$-) models and whose arrows are called ( $\Sigma$-)morphisms.

4. a relation $=_{\Sigma} \subseteq|\operatorname{Mod}(\Sigma)| \times \operatorname{Sen}(\Sigma)$ for each $\Sigma \in \mid$ Sign $\mid$, called ( $\Sigma$-) satisfaction, such that for each morphism $\varphi: \Sigma \rightarrow \Sigma^{\prime}$ in Sign, the satisfaction condition

$$
M^{\prime} \models_{\Sigma^{\prime}} \operatorname{Sen}(\varphi)(e) \text { iff }\left.\operatorname{Mod}(\varphi)\left(M^{\prime}\right)\right|_{\Sigma} e
$$

holds for all $M^{\prime} \in\left|\operatorname{Mod}\left(\Sigma^{\prime}\right)\right|$ and $e \in \operatorname{Sen}(\Sigma)$. Following the usual notational conventions, we sometimes let $1_{\varphi}$ denote the reduct functor $\operatorname{Mod}(\varphi)$ and let $\varphi$ denote the sentence translation $\operatorname{Sen}(\varphi)$. When $M=M^{\prime} 1_{\varphi}$ we say that $M^{\prime}$ is a $\varphi$-expansion of $M$, and that $M$ is the $\varphi$-reduct of $M^{\prime}$; and similarly for model morphisms.

For all the following concepts related to institutions that we recall below, the reader is referred to [14] unless some other place is explicitly indicated.

Let $\Sigma$ be a signature. Then,

- for each $E \subseteq \operatorname{Sen}(\Sigma)$, let $E^{*}=\left\{M \in|\operatorname{Mod}(\Sigma)||M|_{\Sigma} e\right.$ for all $\left.e \in E\right\}$.

- for each class $\mathcal{M}$ of $\Sigma$-models, let $\mathcal{M}^{*}=\left\{e \in \operatorname{Sen}(\Sigma) \mid M \models_{\Sigma} e\right.$ for all $M \in \mathcal{M}\}$.

With no danger of confusion, we let $\bullet$ denote any of the two compositions $* *$ of the two operators $*$. Each of the two bullets is a closure operator. When $E$ and $E^{\prime}$ are sets of sentences of the same signature $\Sigma$, we let $E \models_{\Sigma} E^{\prime}$ denote the fact that $E^{*} \subseteq E^{\prime *}$. The relation $\models_{\Sigma}$ between sets of sentences is called the $(\Sigma$-) semantic consequence relation (notice that it is written just like the satisfaction relation). If $E^{\prime}=\left\{e^{\prime}\right\}$, we might write $E \models_{\Sigma} e^{\prime}$. In order to simplify notation, we usually write $\models$ instead of $\models_{\Sigma}$, for both the satisfaction relation and the semantic consequence relation. Two sentences $e$ and $e^{\prime}$ are called equivalent, denoted $e \equiv e^{\prime}$, if $\{e\}^{*}=\left\{e^{\prime}\right\}^{*}$. Dually, two models $M$ and $M^{\prime}$ are called elementary equivalent, denoted $M \equiv M^{\prime}$, if $\{M\}^{*}=\left\{M^{\prime}\right\}^{*}$.

An institution is called semi-exact [22] if the model functor Mod : Sign $\rightarrow$ Cat ${ }^{o p}$ preserves pushouts. Semi-exactness implies the following amalgamation property for any pushout of signature morphisms $\left(\Sigma_{2} \stackrel{\varphi_{2}}{\longleftarrow} \Sigma \stackrel{\varphi_{1}}{\longrightarrow} \Sigma_{1}, \Sigma_{2} \stackrel{\varphi_{1}^{\prime}}{\longrightarrow} \Sigma^{\prime} \stackrel{\varphi_{2}^{\prime}}{\longleftarrow} \Sigma_{1}\right)$ : for any $M_{1} \in\left|\operatorname{Mod}\left(\Sigma_{1}\right)\right|, M_{2} \in\left|\operatorname{Mod}\left(\Sigma_{2}\right)\right|$ such that $M_{1} 1_{\varphi_{1}}=M_{2} 1_{\varphi_{2}}$, there exists a unique model $M^{\prime} \in\left|\operatorname{Mod}\left(\Sigma^{\prime}\right)\right|$ such that $M^{\prime} 1_{\varphi_{2}^{\prime}}=M_{1}$ and $M^{\prime} 1_{\varphi_{1}^{\prime}}=M_{2}$. An 
analogous property is implied about model morphisms. An institution is called liberal on signature morphisms if the functor $\operatorname{Mod}(\varphi)$ has a left adjoint for each signature morphism $\varphi$.

A presentation is a pair $(\Sigma, E)$, where $E \subseteq \operatorname{Sen}(\Sigma)$. A theory is a presentation $(\Sigma, E)$ with $E$ closed, i.e., with $E^{\bullet}=E$. One usually calls "presentation" or "theory" only the set $E$, and not the whole pair $(\Sigma, E)$. A presentation morphism $\varphi:(\Sigma, E) \rightarrow\left(\Sigma^{\prime}, E^{\prime}\right)$ is a signature morphism $\varphi: \Sigma \rightarrow \Sigma^{\prime}$ such that $\varphi(E) \subseteq E^{\prime \bullet}$. A presentation morphism between theories is called theory morphism. For a presentation $(\Sigma, E)$, we let $\operatorname{Mod}(\Sigma, E)$ denote the category of all $\Sigma$-models $A$ such that $A=E$.

A sentence $\rho \in \operatorname{Sen}(\Sigma)$ is called basic [7] if there exists a $\Sigma$-model $M_{\rho}$ such that, for all $\Sigma$-models $M, M \models \rho$ iff there exists a morphism $M_{\rho} \rightarrow M$. If, in addition, $M_{\rho}$ is a finitely presented object in $\operatorname{Mod}(\Sigma), \rho$ is called finitary basic [7]. Basic sentences tend to be the starting building blocks for sentences in concrete institutions. For instance, in the institution of first-order predicate logic FOPL (see below the examples of institutions), conjunctions of ground atoms are basic. In this paper, we shall be interested in institutions whose sentences are accessible from basic sentences by means of several first-order constructs; hence it suffices to identify enough basic sentences, like the conjunctions of ground atoms above, in order to ensure accessibility. However, the concept of basic sentence turns out to be quite comprehensive in concrete cases; for instance, existentially quantified atoms are also basic in FOPL. The attribute "finitary" is usually equivalent, in concrete cases, to the property that the sentence has only a finite number of symbols. All basic sentences in FOPL are also finitary basic, because FOPL is a "finitary" logic; this is not the case of the institution of infinitary first-order predicate logic IFOPL, where basic sentences with an infinite number of symbols can be constructed by means of infinite conjunctions of atoms.

The sentences of an institution $\mathcal{I}$ can be naturally extended with first-orderlike constructions [34]: if $\varphi: \Sigma \rightarrow \Sigma^{\prime}, \rho, \delta \in \operatorname{Sen}(\Sigma), \rho^{\prime} \in \operatorname{Sen}\left(\Sigma^{\prime}\right)$, and $E \subseteq$ $\operatorname{Sen}(\Sigma)$, one can build the sentences $\neg \rho, \delta \wedge \rho, \delta \vee \rho, \wedge E, \bigvee E,(\forall \varphi) \rho^{\prime},(\exists \varphi) \overline{\rho^{\prime}}$ by means of negation, conjunction, disjunction, arbitrary conjunction, arbitrary disjunction, universal and existential quantification (over signature morphisms) respectively, with the following semantics for each $\Sigma$-model $M$ :

$-M \models \neg \rho$ iff $M \not \models \rho$;

- $M \models \delta \wedge \rho$ iff $M \models \delta$ and $M \models \rho$;

- $M=\delta \vee \rho$ iff $M \models \delta$ or $M \mid=\rho$;

$-M=\bigwedge E$ iff $M \mid=e$ for each $e \in E$;

$-M \models \bigvee E$ iff $M \models e$ for some $e \in E$;

- $M=(\forall \varphi) \rho^{\prime}$ iff $M^{\prime} \models \rho^{\prime}$ for each $\varphi$-expansion $M^{\prime}$ of $M$;

- $M=(\exists \varphi) \rho^{\prime}$ iff $M^{\prime}=\rho^{\prime}$ for some $\varphi$-expansion $M^{\prime}$ of $M$.

It might be the case that the newly constructed sentences are equivalent to some existing sentences in $\mathcal{I}$. The notion of a class of sentences closed under either one of the above constructions (e.g., under conjunction, or under universal quantification over a morphism $\varphi$ ) should be clear. An institution is said to admit negation if the class of all its sentences is closed under negation. 


\section{Examples of institutions}

1. FOPL - the institution of (many-sorted) first-order predicate logic (with equality). The signatures are triplets $(S, F, P)$, where $S$ is the set of sorts, $F=\left\{F_{w, s}\right\}_{w \in S^{*}, s \in S}$ is the $\left(S^{*} \times S\right.$-indexed) set of operation symbols, and $P=\left\{P_{w}\right\}_{w \in S^{*}}$ is the $\left(S^{*}\right.$-indexed) set of relation symbols. If $w=\lambda$, an element of $F_{w, s}$ is called a constant symbol, or a constant. By a slight notational abuse, we let $F$ and $P$ also denote $\bigcup_{(w, s) \in S^{*} \times S} F_{w, s}$ and $\bigcup_{w \in S^{*}} P_{w}$ respectively. A signature morphism between $(S, F, P)$ and $\left(S^{\prime}, F^{\prime}, P^{\prime}\right)$ is a triplet $\varphi=\left(\varphi^{\text {sort }}, \varphi^{o p}, \varphi^{\text {rel }}\right)$, where $\varphi^{\text {sort }}: S \rightarrow S^{\prime}, \varphi^{o p}: F \rightarrow F^{\prime}, \varphi^{\text {rel }}: P \rightarrow$ $P^{\prime}$ such that $\varphi^{o p}\left(F_{w, s}\right) \subseteq F_{\varphi^{\text {sort }}(w), \varphi^{\text {sort }}(s)}^{\prime}$ and $\varphi^{\text {rel }}\left(P_{w}\right) \subseteq P_{\varphi^{\text {sort }}(w)}^{\prime}$ for all $(w, s) \in S^{*} \times S$. When there is no danger of confusion, we may let $\varphi$ denote each of $\varphi^{\text {sort }}, \varphi^{\text {rel }}$ and $\varphi^{o p}$. Given a signature $\Sigma=(S, F, P)$, a $\Sigma$-model $A$ is a triplet $A=\left(\left\{A_{s}\right\}_{s \in S},\left\{A_{w, s}(\sigma)\right\}_{(w, s) \in S^{*}} \times S, \sigma \in F_{w, s},\left\{A_{w}(R)\right\}_{w \in S^{*}, R \in P_{w}}\right)$ interpreting each sort $s$ as a set $A_{s}$, each operation symbol $\sigma \in F_{w, s}$ as a function $A_{w, s}(\sigma): A^{w} \rightarrow A_{s}$ (where $A^{w}$ stands for $A_{s_{1}} \times \ldots \times A_{s_{n}}$ if $w=s_{1} \ldots s_{n}$ ), and each relation symbol $R \in P_{w}$ as a relation $A_{w}(R) \subseteq A^{w}$. When there is no danger of confusion we may let $A_{\sigma}$ and $A_{R}$ denote $A_{w, s}(\sigma)$ and $A_{w}(R)$ respectively. Morphisms between models are the usual $\Sigma$-homomorphisms, i.e., $S$-sorted functions that preserve the structure. The $\Sigma$-sentences are obtained from atoms, i.e., equality atoms $t_{1}=t_{2}$, where $t_{1}, t_{2} \in\left(T_{F}\right)_{s},{ }^{2}$ or relational atoms $R\left(t_{1}, \ldots, t_{n}\right)$, where $R \in P_{s_{1} \ldots s_{n}}$ and $t_{i} \in\left(T_{F}\right)_{s_{i}}$ for each $i \in\{1, \ldots, n\}$, by applying for a finite number of times:

- negation, conjunction, disjunction;

- universal or existential quantification over finite sets of constants (variables).

Satisfaction is the usual first-order satisfaction and is defined using the natural interpretations of ground terms $t$ as elements $A_{t}$ in models $A$. The definitions of functors Sen and Mod on morphisms are the natural ones: for any signature morphism $\varphi: \Sigma \rightarrow \Sigma^{\prime}, \operatorname{Sen}(\varphi): \operatorname{Sen}(\Sigma) \rightarrow \operatorname{Sen}\left(\Sigma^{\prime}\right)$ translates sentences symbolwise, and $\operatorname{Mod}(\varphi): \operatorname{Mod}\left(\Sigma^{\prime}\right) \rightarrow \operatorname{Mod}(\Sigma)$ is the forgetful functor.

2. $(\Pi \cup \Sigma)_{n}^{0}$ - the fragment of $F O P L$ containing only sentences that are equivalent to sentences in prenex normal form that have at most $n$ alternated blocks of quantifiers (universal and existential). Within a given signature, the mentioned set of sentences actually puts together two well-known types of firstorder sentences: $\Pi_{n}^{0}$ and $\Sigma_{n}^{0}[4]$.

3. PFOPL [6] - the institution of partial first-order predicate logic, an extension of $F O P L$ whose signatures $\Sigma=\left(S, F, F^{\prime}, P\right)$ contain, besides relation and (total) operation symbols (in $F$ and $P$ ), partial operation symbols too, in $F^{\prime}$. Models of course interpret the symbols in $F^{\prime}$ as partial operations of appropriate ranks. $\Sigma$-model morphisms $h: A \rightarrow B$ are $S$-sorted functions which commute with the total operations and relations in the usual way, and with the partial operations $\sigma \in F_{s_{1} \ldots s_{n}, s}^{\prime}$ in the following way: for each $\left(a_{1}, \ldots, a_{n}\right) \in A_{s_{1} \ldots s_{n}}$, if

\footnotetext{
${ }^{2} T_{F}$ is the ground term algebra over $F$.
} 
$A_{\sigma}\left(a_{1}, \ldots, a_{n}\right)$ is defined, then so is $B_{\sigma}\left(h_{s_{1}}\left(a_{1}\right), \ldots, h_{s_{n}}\left(a_{n}\right)\right)$, and in this case the latter is equal to $h_{s}\left(A_{\sigma}\left(a_{1}, \ldots, a_{n}\right)\right)$. A signature morphism between $\left(S, F, F^{\prime}, P\right)$ and $\left(S_{0}, F_{0}, F_{0}^{\prime}, P_{0}\right)$ is a $F O P L$-signature morphism $\varphi:\left(S, F \cup F^{\prime}, P^{\prime}\right) \rightarrow\left(S_{0}, F_{0} \cup\right.$ $\left.F_{0}^{\prime}, P_{0}^{\prime}\right)$ such that, for each $\sigma \in F$, it holds that $\varphi^{o p}(\sigma) \in F_{0}$. Thus signature morphisms are allowed to map partial operation symbols to total operation symbols, but not vice versa. There exist two kinds of atoms: (existential) equality atoms $t=t^{\prime}$ and relational atoms $R\left(t_{1}, \ldots, t_{n}\right)$, having syntax just like at FOPL. An equality atom $t=t^{\prime}$ holds in a model $A$ when both terms are defined and equal $\left(A_{t}=A_{t^{\prime}}\right)$. A relational atom $R\left(t_{1}, \ldots, t_{n}\right)$ holds when all terms $t_{i}$ are defined and their interpretations $A_{t_{i}}$ stay in relation $A_{R}$. The sentences are obtained from atoms just like in the case of FOPL (quantification over variables is allowed in the usual sense, which corresponds to considering the quantified variables as new total constants). Note that other kinds of sentences usually considered in partial algebraic frameworks can be expressed here: definedness, $t \downarrow$, as $t=t$; strong equality, $t={ }_{s} t^{\prime}$ (either both $t$ and $t^{\prime}$ are undefined, or both are defined and equal), as $\left(\neg t \downarrow \wedge \neg t^{\prime} \downarrow\right) \vee t=t^{\prime}$; weak equality, $t={ }_{w} t^{\prime}$ (if both $t$ and $t^{\prime}$ are defined, then they are equal), as $(\neg t \downarrow) \vee\left(\neg t^{\prime} \downarrow\right) \vee t=t^{\prime}$. The functor Mod is defined similarly to the case of FOPL.

4. $P A$ - the institution of partial algebra, a fragment of $P F O P L$ having signatures without relation symbols. Partial algebras and their applications were extensively studied in [28] and [3].

5. IFOPL - the institution of infinitary first-order logic, an infinitary extension of FOPL, which allows conjunction on arbitrary sets of sentences. This logical system is known under the name $L_{\infty, \omega}[21,20]$ and plays an important role in categorical logic.

6. IFOPL $L_{\alpha}$ (where $\alpha$ is an infinite cardinal) - a fragment of IFOPL, admitting only conjunction on sets of sentences with cardinal smaller than $\alpha$. This logical system is usually called $L_{\alpha, \omega}$ [18]. Note that $I F O P L_{\omega}$ is $F O P L$.

7. PosFOPL - the institution of positive first order predicate logic, a fragment of $F O P L$, with sentences constructed by means of $\wedge, \vee, \forall, \exists$, but not negation $\neg$. Here $\vee$ and $\exists$ are no longer reducible to $\wedge$ and $\forall$ or vice versa. Positive sentences are defined and studied for example in $[4,25]$.

8. EQL - the institution of equational logic [14], a fragment of FOPL, with no relation (only operation) symbols, and with sentences constructed from atoms only by means of universal quantification (no logical connectives).

9. $E Q L N$ - a minimal extension of $E Q L$ with negation, allowing sentences obtained from atoms and negations of atoms through only one round of quantification, either universal or existential, over a set of variables. More precisely, all sentences have the form $(Q X) t_{1} k t_{2}$, where $Q \in\{\forall, \exists\}$ and $k \in\{=, \neq\}$. Note that this institution admits negation.

10. $R W L$ - the institution of (unconditional) rewriting logic. It has the same signatures as $E Q L$, but models have in addition a preorder relation on each sort carrier, compatible with the operations, and model morphisms have to be increasing w.r.t. these preorders. The sentences are usual equations as in $E Q L$ and transitions $(\forall X) t \rightarrow t^{\prime}$, with $\rightarrow$ interpreted as the model preorder. This 
logic cannot be seen as a fragment of FOPL, due to the built-in nature of the preorder on models. Rewriting logic was introduced in [23] with models having a categorical structure where arrows express different transitions between states; a simplified and more amenable version of this logic, which forces this categorical structure to be a preorder, is used in specification languages such as CafeObj [11] or Maude [5]; this simplified version was considered here.

11. $O S L$ - the institution of order-sorted (equational) logic [29], an extension of $E Q L$ where each signature has a partial order on the set of sorts. Thus a signature is a triplet $(S, \leq, F)$, where $(S, \leq)$ is a partially ordered set and $(S, F)$ is an $E Q L$-signature. A $(S, \leq, F)$-model is an $(S, F)$-model in $E Q L$ subject to two additional requirements:

- for each $s, s^{\prime} \in S$ with $s \leq s^{\prime}$, it holds that $A_{s} \subseteq A_{s^{\prime}}$;

- for each $(w, s),\left(w^{\prime}, s^{\prime}\right) \in S^{*} \times S$ such that $w=s_{1} \ldots s_{n}, w^{\prime}=s_{1}^{\prime} \ldots s_{n}^{\prime}, s_{i} \leq$ $s_{i}^{\prime}$, and $s \leq s^{\prime}$, and each $\sigma \in F_{w, s} \cap F_{w^{\prime}, s^{\prime}}$, it holds that $A_{w, s}(\sigma): A^{w} \rightarrow A_{s}$ restricts and corestricts $A_{w^{\prime}, s^{\prime}}(\sigma): A^{w^{\prime}} \rightarrow A_{s^{\prime}}$.

A $(S, \leq, F)$-morphism between $A$ and $B$ is a $(S, F)$-morphism in $E Q L, h: A \rightarrow$ $B$, such that for all $s, s^{\prime} \in S$ with $s \leq s^{\prime}$, it holds that $h_{s}$ restricts and corestricts $h_{s^{\prime}}$. Given a signature $\Sigma=(S, \leq, F)$, one can construct the ground term $\Sigma$ algebra $T_{\Sigma}$ similarly to the case of $E Q L$, just that one needs to consider the subsort relationship $\leq$ too. An $(S, \leq, F)$-sentence is an equation $(\forall X) t=t^{\prime}$, where $X$ is an $S$-sorted set of variables and $t, t^{\prime} \in T_{(S, \leq, F \cup X)} \cdot{ }^{3}$ Satisfaction of a sentence by a model is defined in the obvious way. The functor Mod acts just like in the case of $E Q L$.

12. $M L$ - the institution of (unconditional) membership equational logic, an extension of $E Q L$ which calls the usual sorts "kinds", and allows on each kind a set of sorts that are to be interpreted, on models, as subsets of the kind carrier. Thus a signature is a triplet $\left(K,\left\{S_{k}\right\}_{k \in K}, F\right)$, where $(K, F)$ is an $E Q L$-signature and for each kind $k \in K, S_{k}$ is the set of sorts for this kind. Besides equations, this logic also has membership assertions: $(\forall X) t: s$, where $t \in\left(T_{\Sigma}(X)\right)_{k}$ and $s \in S_{k}$, meaning that " $t$ is of sort $s$ ". This logic, introduced in [24], can be seen as a fragment of FOPL which only uses unary relation symbols and has only universally quantified atoms as sentences. As shown in [24], $M L$ naturally embeds (a variation of) OSL.

13. $E H L$ - the institution of extended hidden logic. The signatures are triplets $(H, V, F)$, where:

- $H$ is the set of hidden sorts;

- $V$ is the set of visible sorts, $V \cap H=\emptyset$;

- $(H \cup V, F)$ is an $E Q L$-signature (i.e., $F$ is an $(H \cup V)^{*} \times(H \cup V)$-indexed set of operation symbols).

The $(H, V, F)$-models are the usual $(H \cup V, F)$-models from $E Q L$. For a model $A$, one defines its behavioral equivalence $\equiv_{A}$ to be the least congruence on $A$ which

\footnotetext{
${ }^{3}$ The variables in $X$ are interpreted as new constants.
} 
is an identity on visible sorts. The $(H, V, F)$-morphsism are the $(H \cup V, F)$ morphisms from $E Q L$ that preserve behavioral equivalence. There are two kinds of atoms: (usual) equality atoms $t=t^{\prime}$ and behavioral equality atoms $t \equiv t^{\prime}$. Satisfaction of equality atoms is the usual first-order satisfaction. For a $(H, V, F)$ model $A, A=t \equiv t^{\prime}$ iff $A_{c\left[x_{1} \leftarrow a_{1}, \ldots, x_{n} \leftarrow a_{n}, z \leftarrow t\right]}=A_{c\left[x_{1} \leftarrow a_{1}, \ldots, x_{n} \leftarrow a_{n}, z \leftarrow t^{\prime}\right]}$ for each sort $v \in V$, each sequence $x_{1}, \ldots, x_{n}$ of variables of various sorts, each context $c$ in $T_{F}\left(\left\{x_{1}, \ldots, x_{n}, z\right\}\right)$ of sort $v$, and each sequence $a_{1}, \ldots, a_{n}$ of elements in $A$ of appropriate sorts. (Here a context is a term with only one occurrence of the variable $z ; z$ is assumed to have the same sort as $t$ and $t^{\prime}$. Also, for instance $c\left[x_{1} \leftarrow\right.$ $\left.a_{1}, \ldots, x_{n} \leftarrow a_{n}, z \leftarrow t\right]$ is a ground $(H \cup V, F)$-term parameterized by elements in $A$, and $A_{c\left[x_{1} \leftarrow a_{1}, \ldots, x_{n} \leftarrow a_{n}, z \leftarrow t\right]}$ is its natural interpretation as an element in $A$.) All sentences are constructed from atoms by means of first-order connectives and quantifiers just like in the case of $F O P L$ (quantification is allowed over variables of both hidden and visible sorts). A signature morphism between $(H, V, F)$ and $\left(H^{\prime}, V^{\prime}, F^{\prime}\right)$ is an $E Q L$-signature morphism $\varphi:(H \cup V, F) \rightarrow\left(H^{\prime} \cup V^{\prime}, F^{\prime}\right)$ such that:

$-\varphi(V) \subseteq V^{\prime}, \varphi(H) \subseteq H^{\prime}$

- for each $\left(w^{\prime}, s^{\prime}\right) \in\left(H^{\prime} \cup V^{\prime}\right)^{*} \times\left(H^{\prime} \cup V^{\prime}\right)$ such that $w^{\prime}$ contains a sort in $\varphi^{\text {sort }}(H)$, and $\sigma^{\prime} \in F_{w^{\prime}, s^{\prime}}^{\prime}$, there exists $\sigma \in F$ such that $\varphi^{o p}(\sigma)=\sigma^{\prime}$.

On signature morphisms, the functors Mod and Sen act as in the case of $E Q L$. The above description of $E H L$ was adapted from [8]. See [15] for details about hidden logic, and [2] for the description of (a variation of) full first-order hidden logic.

14. $H L$ - $[16,12]$ the institution of hidden logic, a fragment of $E H L$, with sentences constructed from atoms only by means of universal quantification (no logical connectives).

\section{Representable and quasi-representable signature morphisms}

The institutional notions of representable and quasi-representable signature morphisms are abstract concepts meant to capture the phenomena of quantification over (sets of) first-order variables. Both notions start from the fact that semantics of quantification in first-order-like logics can be given in terms of signature extensions: $M \models_{(S, F, P)}(\forall X) e\left(M \models_{(S, F, P)}(\exists X) e\right)$ iff $M^{\prime} \models_{(S, F \cup X, P)} e$ for each (for some) ( $S, F \cup X, P)$-expansion $M^{\prime}$ of $M$. Thus, in order to reach first-order quantification institutionally, one needs to define somehow what "injective signature morphism that only adds constant symbols" (such as $\iota:(S, F, P) \rightarrow$ $(S, F \cup X, P))$ means.

Definition 2 A signature morphism $\varphi: \Sigma \rightarrow \Sigma^{\prime}$ is called:

- representable [7], if there exists a $\Sigma$-model $M_{\varphi}$ (called the representation of $\varphi)$ and an isomorphism of categories $I_{\varphi}: \operatorname{Mod}\left(\Sigma^{\prime}\right) \rightarrow M_{\varphi} / \operatorname{Mod}(\Sigma)$ such that $I_{\varphi} ; U=\operatorname{Mod}(\varphi)$, where $U: M_{\varphi} / \operatorname{Mod}(\Sigma) \rightarrow \operatorname{Mod}(\Sigma)$ is the usual forgetful functor; 
- finitely representable [7], if it is representable and $M_{\varphi}$ is a finitely presented object in $\operatorname{Mod}(\Sigma)$;

- quasi-representable [6], if for each $A^{\prime} \in\left|\operatorname{Mod}\left(\Sigma^{\prime}\right)\right|$, the canonical functor $A^{\prime} / \operatorname{Mod}(\varphi): A^{\prime} / \operatorname{Mod}\left(\Sigma^{\prime}\right) \rightarrow A^{\prime} 1_{\varphi} / \operatorname{Mod}(\Sigma)$ is an isomorphism of categories;

- finitely quasi-representable [6], if it is quasi-representable and for each colimit $\left(A_{i} \stackrel{\mu_{i}}{\rightarrow} A\right)_{i \in I}$ of a directed diagram of $\Sigma$-models $\left(A_{i} \stackrel{h_{i, j}}{\rightarrow} A_{j}\right)_{i, j \in I, i \leq j}$ and each $\varphi$-expansion $A^{\prime}$ of $A$, there exists an index $i \in I$ and a $\varphi$-expansion $\mu_{i}^{\prime}$ of $\mu_{i}$.

The notion of representability is built on the intuition that, in FOPL, an expansion of a $\Sigma=(S, F, P)$-model $A$ over a signature inclusion $\iota: \Sigma \rightarrow \Sigma^{\prime}=$ $(S, F \cup X, P)$ that only adds constants can be viewed as a pair $(M, v)$, where $v: X \rightarrow M$ is a function interpreting the new constants in $X$, and furthermore as a pair $(M, \bar{v})$, where $\bar{v}: T_{\Sigma}(X) \rightarrow M$ is a model-morphism. ${ }^{4}$ Hence $\iota$ is represented by $T_{\Sigma}(X)$. And $\iota$ is finitely representable, i.e., $T_{\Sigma}(X)$ is finitely presented in $\operatorname{Mod}(\Sigma)$, if $X$ is finite.

On the other hand, quasi-representability follows the intuition that the above signature inclusion $\iota$ does not allow multiple expansions of $\Sigma$-morphisms $A \stackrel{h}{\rightarrow} B$ having a fixed source $A^{\prime}$ (where $A^{\prime}$ is a $\iota$-expansion of $A$ ). This is because $A^{\prime}$ already "indicates", via $h$, how the constants in $X$ should be interpreted in the target model $B^{\prime}$ of a presumptive $\iota$-expansion $h^{\prime}$ of $h$; and of course $h^{\prime}$ has to be identical, as a function, to $h$. Thus $\iota$ is also quasi-representable. And again, $\iota$ is finitely quasi-represented if $X$ is finite. Intuitively, if we regard directed colimits of $\Sigma$-models as "unions", all the interpretations in the "union model" $A$ of the finite number of constants in $X$ will eventually be reached by one of the members $A_{i}$ of the union; hence the "inclusion" of $A_{i}$ into $A$ has a $\iota$-expansion.

For most concrete institutions (at least for those admitting initial objects in the categories of models, like our examples 1-14), the notions of representability and quasi-representability coincide, as shown by the below lemma.

Lemma 3 [6] Let $\varphi: \Sigma \rightarrow \Sigma^{\prime}$ be a signature morphism. Then $[\varphi$ is (finitely) quasirepresentable and $\operatorname{Mod}(\Sigma)$ has an initial object] iff $\varphi$ is (finitely) representable.

It is shown in [6] that quasi-representable signature morphisms create directed colimits. Throughout the paper, we are going to use intensively a similar property:

Lemma 4 Let $\varphi: \Sigma \rightarrow \Sigma^{\prime}$ be a quasi-representable signature morphism and let $\left(A_{i} \stackrel{f_{i, j}}{\rightarrow} A_{j}\right)_{i, j \in I, i \leq j}$ be a directed diagram in $\operatorname{Mod}(\Sigma)$ with colimit $\left(A_{i} \stackrel{\mu_{i}}{\rightarrow} A\right)_{i \in I}$. Also, let $k \in I, I_{k}=\{i \in I \mid k \leq i\}$, and $B$ a $\varphi$-expansion of $A_{k}$. Then there exists a directed diagram $\left(A_{i}^{\prime} \stackrel{f_{i, j}^{\prime}}{\rightarrow} A_{j}^{\prime}\right)_{i, j \in I_{k}, i \leq j}$ in $\operatorname{Mod}\left(\Sigma^{\prime}\right)$, with colimit $\left(A_{i}^{\prime} \stackrel{\mu_{i}^{\prime}}{\rightarrow} A^{\prime}\right)_{i \in I_{k}}$, such that:

\footnotetext{
${ }^{4} T_{\Sigma}(X)$ is the term algebra over variables $X$ and operations in $F$, with all relations
} in $P$ empty. 
$-A_{k}^{\prime}=B$

- for each $i, j \in I_{k}$ with $i \leq j, f_{i, j}^{\prime}$ is a $\varphi$-expansion of $f_{i, j}$;

- for each $i \in I_{k}, \mu_{i}^{\prime}$ is a $\varphi$-expansion of $\mu_{i}$.

Proof:

For each $i \in I_{k}$, we define $\left(f_{k, i}^{\prime}: B \rightarrow A_{i}^{\prime}, A_{i}^{\prime}\right)$ to be $\left(A_{k} / \operatorname{Mod}(\varphi)\right)^{-1}\left(f_{k, i}:\right.$ $\left.A_{k} \rightarrow A_{i}, A_{i}\right)$. In particular, for $i=k$, we have $A_{k}^{\prime}=B$ and $f_{k, k}^{\prime}=1_{B}$. Also, for each $i, j \in I_{k}$ with $k<i \leq j$, we define $f_{i, j}^{\prime}$ to be $\left(A_{k} / \operatorname{Mod}(\varphi)\right)^{-1}\left(f_{i, j}\right.$ : $\left.\left(f_{k, i}, A_{i}\right) \rightarrow\left(f_{k, j}, A_{j}\right)\right)$. Then $D g^{\prime}=\left(A_{i}^{\prime} \stackrel{f_{i, j}^{\prime}}{\rightarrow} A_{j}^{\prime}\right)_{i, j \in I_{k}, i \leq j}$ is a directed diagram in $\operatorname{Mod}\left(\Sigma^{\prime}\right)$ and its $\varphi$-reduct is $D g=\left(A_{i} \stackrel{f_{i, j}}{\rightarrow} A_{j}\right)_{i, j \in I_{k}, i \leq j}$. Now we define $\left(\mu_{k}^{\prime}\right.$ : $\left.B \rightarrow A^{\prime}, A^{\prime}\right)$ to be $\left(A_{k} / \operatorname{Mod}(\varphi)\right)^{-1}\left(\mu_{k}: A_{k} \rightarrow A, A\right)$ and, for each $i \in I_{k}, \mu_{i}^{\prime}$ to be $\left(A_{k} / \operatorname{Mod}(\varphi)\right)^{-1}\left(\mu_{i}:\left(f_{k, i}, A_{i}\right) \rightarrow\left(\mu_{k}, A\right)\right)$. Then $C C^{\prime}=\left(A_{i}^{\prime} \stackrel{\mu_{i}^{\prime}}{\rightarrow} A^{\prime}\right)_{i \in I_{k}}$ is a cocone of $D g^{\prime}$, and the $\varphi$-reduct of $C C^{\prime}$ is $C C=\left(A_{i} \stackrel{\mu_{i}}{\rightarrow} A\right)_{i \in I_{k}}$. The fact that $C C^{\prime}$ is an actual colimit for $D g^{\prime}$ follows at once by the quasi-representability of $\varphi$ : for any cocone $\left(A_{i}^{\prime} \stackrel{\nu_{i}^{\prime}}{\rightarrow} A^{\prime \prime}\right)_{i \in I_{k}}$ of $D g^{\prime}$, we get that its reduct $\left(A_{i}^{\prime} 1_{\varphi} \stackrel{\nu_{i}^{\prime} 1_{\varphi}}{\rightarrow} A^{\prime \prime} 1_{\varphi}\right)_{i \in I_{k}}$ is a cocone of $D g$; thus if one takes $u$ to be the universal arrow from $C C$ to $\left(A_{i}^{\prime} 1_{\varphi} \stackrel{\left.\nu_{i}^{\prime}\right|_{\varphi}}{\longrightarrow} A^{\prime \prime} 1_{\varphi}\right)_{i \in I_{k}}$ (according to Lemma 1), then $\left(A_{k} / \operatorname{Mod}(\varphi)\right)^{-1}\left(u:\left(\mu_{k}, A\right) \rightarrow\right.$ $\left.\left(\nu_{k}^{\prime} 1_{\varphi}, A^{\prime \prime} 1_{\varphi}\right)\right)$ is the desired universal arrow from $C C^{\prime}$ to $\left(A_{i}^{\prime} \stackrel{\nu_{i}^{\prime}}{\rightarrow} A^{\prime \prime}\right)_{i \in I_{k}}$. q.e.d.

The next lemma shows that (quasi-)representable signature morphisms behave well under composition and pushouts.

Lemma $5[6]$

1. (Finitely) quasi-representable signature morphisms form a subcategory of Sign.

2. (Finitely) representable signature morphisms form a subcategory of Sign.

3. If the institution is semi-exact, then the class of (finitely) quasi-representable signature morphisms is closed under pushouts.

4. If the institution is semi-exact and liberal on signature morphisms, then the class of (finitely) representable signature morphisms is closed under pushouts.

Of course, representability and quasi-representability are only abstract approximations for "injective morphisms that only add constants". What will be relevant for the results of this paper is that in all our examples 1-14 of institutions, (quasi-)representable signature morphism include the desired types of morphisms. Formally, let $\varphi: \Sigma \rightarrow \Sigma^{\prime}$ be a signature morphism in any of the examples 1-14 of institutions. We say that $\varphi$ is an injective signature morphism that only adds constants if the following conditions hold: $\varphi^{\text {sort }}$ is bijective, $\varphi^{\text {rel }}$ is bijective, $\varphi^{o p}$ is injective, and $F^{\prime}-\varphi^{o p}(F)$ contains only (total) operation symbols. (Here, $F$ and $F^{\prime}$ stand for the sets of all (partial and total) operation symbols of $\Sigma$ and $\Sigma^{\prime}$ respectively.) If in addition $F^{\prime}-\varphi^{o p}(F)$ is finite, we say that $\varphi$ is an injective signature morphism that only adds finitely many constants. 
Proposition 6 In any of the examples 1-14 of institutions, all injective signature morphisms that only add (finitely many) constants are (finitely) representable, hence also (finitely) quasi-representable. Moreover, in each case, the (broad) subcategory of Sign of such morphisms is closed under pushouts.

Proof:

The fact that such morphisms are (finitely) representable can be shown using arguments very similar to the ones for FOPL. The only slightly more exotic cases are $P F O P L, P A, O S L, R L, H L$, and $E H L$; however, in each case the algebra freely generated by a set of (total) variables exists in any signature. The cases of $H L$ and $E H L$ actually require a small separate discussion. Let $(H, V, F)$ be a signature in either of the two institutions. Since each free algebra $T_{F}(X)$ has its behavioral equivalence equal to the identity, every $E Q L$-morphism with source $T_{F}(X)$ is also an $E H L$ - and $H L$ - morphism, thus an $E H L$ - or $H L$ - signature morphism that only adds (finitely many) constants $X$ is indeed (finitely) represented by $T_{F}(X)$.

As for closure under pushouts, this follows easily from the fact that, in the category of sets and functions, the subcategory of injective functions is closed under pushouts.

q.e.d.

Although not strictly needed in this paper, but helpful for getting an idea on how close the above mentioned approximation is, we recall a concrete characterization of representable (and quasi-representable) signature morphisms in FOPL.

Proposition 7 [33] Let $\varphi: \Sigma=(S, F, P) \rightarrow \Sigma^{\prime}=\left(S^{\prime}, F^{\prime}, P^{\prime}\right)$ be a signature morphism in FOPL. Then the following are equivalent:

1. $\varphi$ is representable;

2. $\varphi$ is quasi-representable;

3. $\varphi^{\text {sort }}$ and $\varphi^{\text {rel }}$ are bijective, and for all $\left(w^{\prime}, s^{\prime}\right) \in S^{\prime *} \times S^{\prime}$ with $w^{\prime} \neq \lambda$, for all $\sigma^{\prime} \in F_{w^{\prime}, s^{\prime}}^{\prime}$, there exists a unique $\sigma \in F$ such that $\varphi^{o p}(\sigma)=\sigma^{\prime}$ (in other words, $\varphi$ is bijective w.r.t. all items except constant symbols).

Moreover, the above three conditions stay equivalent if we add the word "finitely" to the first two and add the requirement that $F^{\prime}-\varphi^{o p}(F)$ be finite to the third.

\section{Elementary Morphisms}

In classical first-order logic [4], an injective model morphism $A \stackrel{h}{\rightarrow} B$ is called an elementary embedding if one of the following equivalent conditions holds:

1. for each formula $e\left(x_{1}, \ldots, x_{n}\right)$ and each sequence $a_{1}, \ldots, a_{n} \in A, A \models$ $e\left(a_{1}, \ldots, a_{n}\right)$ iff $B=e\left(h\left(a_{1}\right), \ldots, h\left(a_{n}\right)\right)$;

2. for each formula $e\left(x_{1}, \ldots, x_{n}\right)$ and each sequence $a_{1}, \ldots, a_{n} \in A, A$ $e\left(a_{1}, \ldots, a_{n}\right)$ implies $B \models e\left(h\left(a_{1}\right), \ldots, h\left(a_{n}\right)\right)$. 
The notion of elementary embedding has an immediate generalization to the many-sorted case FOPL; and it was extended to cope with infinitary first-order logics too $[18,17]$. Our next institutional generalization reads the concept of elementary embedding in the following way: the morphism $h$ preserves sentences in any language extending with constants the original language, regardless of the actual interpretation of these constants. Notice that the two alternative definitions of elementary embeddings listed above are equivalent thanks to the existence of negations in full first-order logic; however, this is not the case in less expressive logics, such as PosFOPL or EQL. We prefer to consider the second variant and interpret elementarity as a sentence preservation property rather than a refinement of elementary equivalence. This subjective choice is motivated by our belief that taking into consideration the direction of the arrow $h$ in the definition is a more fruitful approach. In an institution, the "languages extended with constants" are captured by quasi-representable signature morphisms $\varphi$ having as source the given language/signature, and satisfaction inside such an extended language is captured by usual satisfaction by $\varphi$-expansions. To keep the discussion general and to avoid certain intricacies in the particular cases resulting from considering all quasi-representable, or representable, signature morphisms, we parameterize our definition by a class $\mathcal{Q}$ of quasi-representable signature morphisms. Thus let $A \stackrel{h}{\rightarrow} B$ be a $\Sigma$-morphism. Formulae $e\left(x_{1}, \ldots, x_{n}\right)$ are expressed by usual sentences $e^{\prime}$ in signatures $\Sigma^{\prime}$, where $\varphi: \Sigma \rightarrow \Sigma^{\prime}$ is a signature morphism in $\mathcal{Q}$. Satisfaction of such sentences $e^{\prime} \in \operatorname{Sen}\left(\Sigma^{\prime}\right)$ makes sense in $\varphi$-expansions of $A$ and $B$, expansions which are to be seen as models together with some designated constants. ${ }^{5}$ However, asking that " $A^{\prime} \models e^{\prime}$ implies $B^{\prime} \models e^{\prime \prime}$ " for all $\varphi$-expansions $A^{\prime}$ and $B^{\prime}$ of $A$ and $B$ and for all $e^{\prime} \in \operatorname{Sen}\left(\Sigma^{\prime}\right)$ is not appropriate, since the quoted implication should be required only about constants in $A$ and $B$ connected through $h$; the connection is realized by first considering $\varphi$-expansions $h^{\prime}$ of $h$.

The injectivity assumption in the definition of elementary embedding for classical first-order logic is superfluous. We did not consider it in the above discussion; this could be seen as yet another subjective choice, meant to emphasize once more the idea of sentence preservation, this time to the prejudice of the algebraic property of model embedding. This choice has an important terminological consequence: we define and study "elementary morphisms", and not "elementary embeddings", although the elementary morphisms yield in particular the FOPLelementary embeddings.

For the whole section, we fix an institution $\mathcal{I}$ and a broad subcategory $\mathcal{Q}$ of Sign (i.e., a class of signature morphisms containing all identity morphisms and closed under composition), consisting of quasi-representable signature morphisms. In particular, by taking further mild assumptions on $\mathcal{I}$ such as semiexactness or liberality on signature morphisms, according to Lemma 5, possible choices for $\mathcal{Q}$ are given by either of the following four types of signature mor-

\footnotetext{
${ }^{5}$ Recall from Section 3 the connection between quasi-representable signature morphisms and first-order variables/constants.
} 
phisms: quasi-representable, finitely quasi-representable, representable, finitely representable.

Definition 8 Let $\Sigma$ be a signature. $A \Sigma$-morphism $A \stackrel{h}{\rightarrow} B$ is called $\mathcal{Q}$-elementary if for all signature morphisms $\varphi: \Sigma \rightarrow \Sigma^{\prime}$ in $\mathcal{Q}, \varphi$-expansions $A^{\prime} \stackrel{h^{\prime}}{\rightarrow} B^{\prime}$ of $h$, and sentences $e^{\prime} \in \operatorname{Sen}\left(\Sigma^{\prime}\right)$, it holds that $A^{\prime}=e^{\prime}$ implies $B^{\prime} \models e^{\prime}$.

\section{Remark 9}

1. Because each morphism in $\mathcal{Q}$ is quasi-representable, in Definition 8 the $\varphi$ expansion $h^{\prime}$ of $h$ is uniquely determined by the $\varphi$-expansion $A^{\prime}$ of $A$.

2. If the institution admits negations, then the condition in Definition 8 can be equivalently stated by replacing " $A^{\prime} \models e^{\prime}$ implies $B^{\prime} \models e^{\prime}$ " with " $A^{\prime} \models e^{\prime}$ iff $B^{\prime}=e^{\prime \prime}$.

Let us see what our general concept of $\mathcal{Q}$-elementary morphism becomes for our examples 1-14 of institutions. In what follows, for all these institutions, we shall simply call elementary morphisms the $\mathcal{Q}$-elementary morphisms with $\mathcal{Q}$ being the category of injective signature morphisms that only add constants. (Note that in logics with finite sentences, such as FOPL and all its fragments, elementarity w.r.t. arbitrary signature morphisms that only add constants is equivalent to elementarity w.r.t. morphisms that only add finitely many constants; this is because just a finite set of the newly added constants are contained in a given sentence.) Known and relatively well-studied cases are the following:

- for FOPL, the elementary embeddings from (the many-sorted version of) classical model theory [4];

- for $P A$, the elementary embeddings of partial algebras [3];

- for IFOPL and IFOP $L_{\alpha}$, the $L_{\infty, w^{-}}$and $L_{\alpha, w^{-}}$elementary embeddings from infinitary model theory $[18,21,17]$;

- for $(\Pi \cup \Sigma)_{1}^{0}$, the existentially closed embeddings [17];

- for $(\Pi \cup \Sigma)_{n}^{0}$, the $\Sigma_{n}^{0}$-extensions [4].

Up to our knowledge, elementary embeddings for the other examples of institutions were not considered so far in the literature. However, such notions are meaningful instances of the logic-independent concept of elementary morphism that we propose here. In each case, an elementary morphism is one that preserves satisfaction of all sentences with elements of the source model as parameters. The next proposition gives some expected properties of $\mathcal{Q}$-elementary morphisms.

Proposition 10 Let $\chi: \Sigma \rightarrow \Sigma^{\prime}$ be a signature morphism. Then the following hold:

1. The $\mathcal{Q}$-elementary morphisms in $\operatorname{Mod}(\Sigma)$ form a subcategory of $\operatorname{Mod}(\Sigma)$.

2. Assume that the institution has pushouts of signatures and is semi-exact, and that $\mathcal{Q}$ is closed under pushouts. If $A^{\prime} \stackrel{h^{\prime}}{\rightarrow} B^{\prime}$ is a $\mathcal{Q}$-elementary morphism in $\operatorname{Mod}\left(\Sigma^{\prime}\right)$, then $h^{\prime} 1_{\chi}$ is also $\mathcal{Q}$-elementary; 
3. If $\chi$ is in $\mathcal{Q}, A \stackrel{h}{\rightarrow} B$ is a $\mathcal{Q}$-elementary morphism in $\operatorname{Mod}(\Sigma)$, and $A^{\prime} \stackrel{h^{\prime}}{\rightarrow} B^{\prime}$ is a $\chi$-expansion of $h$, then $h^{\prime}$ is also $\mathcal{Q}$-elementary.

Proof:

1: If $A \in|\operatorname{Mod}(\Sigma)|$, then $1_{A}$ is $\mathcal{Q}$-elementary because any expansion of $1_{A}$ along a quasi-representable signature morphism is an identity model-morphism itself. Let now $A \stackrel{h}{\rightarrow} B$ and $B \stackrel{g}{\rightarrow} C$ be two $\mathcal{Q}$-elementary morphisms in $\operatorname{Mod}(\Sigma)$ and let $f=h ; g$. In order to show that $f$ is $\mathcal{Q}$-elementary, let $\varphi: \Sigma \rightarrow \Sigma_{0}$ be a signature morphism in $\mathcal{Q}$ and $A_{0} \stackrel{f_{0}}{\rightarrow} C_{0}$ a $\varphi$-expansion of $f$. Let $h_{0}: A_{0} \rightarrow B_{0}$ such that $\left(A_{0}, h_{0}\right)=\left(A_{0} / \operatorname{Mod}(\varphi)\right)^{-1}(A, h)$. Let $g_{0}=\left(A_{0} / \operatorname{Mod}(\varphi)\right)^{-1}(g:(A, h) \rightarrow(A, f))$. Then $h_{0} ; g_{0}=f_{0}$. Since $h$ and $g$ are $\mathcal{Q}$-elementary, it follows that $\left\{A_{0}\right\}^{*} \subseteq$ $\left\{B_{0}\right\}^{*} \subseteq\left\{C_{0}\right\}^{*}$.

2: Let $A \stackrel{h}{\rightarrow} B$ denote the $\chi$-reduct of $h^{\prime}$. In order to prove $h$ elementary, let $\varphi: \Sigma \rightarrow \Sigma_{0}$ be a signature morphism in $\mathcal{Q}$ and $A_{0} \stackrel{h_{0}}{\rightarrow} B_{0}$ a $\varphi$-expansion of $h$. Consider the pushout $\Sigma^{\prime} \stackrel{\varphi^{\prime}}{\rightarrow} \Sigma_{1} \stackrel{\chi^{\prime}}{\leftarrow} \Sigma_{0}$ of the signature morphism span $\left(\Sigma^{\prime} \underset{\chi}{\leftarrow} \Sigma \stackrel{\varphi}{\rightarrow} \Sigma_{0}\right)$. Then $\varphi^{\prime}$ is also in $\mathcal{Q}$. By semi-exactness, since $h^{\prime}$ and $h_{0}$ have a common reduct (that is, $h$ ), they also have a common expansion $A_{1} \stackrel{h_{1}}{\rightarrow} B_{1}$ in $\operatorname{Mod}\left(\Sigma_{1}\right)$. Because $h^{\prime}$ is elementary, $\left\{A_{1}\right\}^{*} \subseteq\left\{B_{1}\right\}^{*}$. Finally, using the satisfaction condition, we get $\left\{A_{0}\right\}^{*} \subseteq\left\{B_{0}\right\}^{*}$.

3: Immediate from the definition of $\mathcal{Q}$-elementary morphisms and the fact that $\mathcal{Q}$ is closed under composition.

q.e.d.

\section{Elementary Chain Property}

Throughout this section, we again fix an institution $\mathcal{I}$ and a broad subcategory $\mathcal{Q}$ of Sign consisting of quasi-representable signature morphisms.

A $\mathcal{Q}$-elementary chain is a chain diagram in $\operatorname{Mod}(\Sigma)$ for some signature $\Sigma$, such that all its morphisms are $\mathcal{Q}$-elementary. The elementary chain property (parameterized by $\mathcal{Q}$ and abbreviated $\mathcal{Q}$-ECP) asks that, for each colimit of each $\mathcal{Q}$-elementary chain, all the structural morphisms be $\mathcal{Q}$-elementary. In other words, it asks that for each signature $\Sigma$, the subcategory of $\operatorname{Mod}(\Sigma)$ of $\mathcal{Q}$ elementary morphisms be closed under chain colimits. We are going to prove that, under appropriate accessibility assumptions on sentences, $\mathcal{Q}$-ECP holds in an arbitrary institution. But first we need to consider some technical concepts and results.

We say that a sentence $e \in \operatorname{Sen}(\Sigma)$ for some signature $\Sigma$ is preserved (reflected) by directed colimits of $\mathcal{Q}$-elementary morphisms, abbreviated $\mathcal{Q}$-preserved (reflected), if for each directed diagram of $\mathcal{Q}$-elementary $\Sigma$-morphisms $\left(A_{i} \stackrel{f_{i, j}}{\rightarrow}\right.$ $\left.A_{j}\right)_{i, j \in I, i \leq j}$ with colimit $\left(A_{i} \stackrel{\mu_{i}}{\longrightarrow} A\right)_{i \in I}$ and each $k \in I, A_{k} \models e$ implies $A=e$ $\left(A \mid=e\right.$ implies $A_{k}=e$ respectively).

Proposition 11 The class of sentences preserved by directed colimits of $\mathcal{Q}$ elementary morphisms 
1. contains all basic sentences,

2. is closed under arbitrary conjunction and disjunction,

3. is closed under existential quantification over morphisms in $\mathcal{Q}$,

4. is closed under universal quantification over finitely quasi-representable morphisms in $\mathcal{Q}$.

Proof:

Let $e \in \operatorname{Sen}(\Sigma),\left(A_{i} \stackrel{f_{i, j}}{\rightarrow} A_{j}\right)_{i, j \in I, i \leq j}$ a directed diagram of $\mathcal{Q}$-elementary $\Sigma$-morphisms, with colimit $\left(A_{i} \stackrel{\mu_{i}}{\longrightarrow} A\right)_{i \in I}$, and let $k \in I$. Assume that $A_{k}=e$. We need to prove that $A \models e$.

1: Assume $e$ is a basic sentence. Since $A_{k} \models e$, there exists a $\Sigma$-morphism $M_{e} \rightarrow A_{k}$, hence, by composition with $\mu_{k}$, we find a morphism $M_{e} \rightarrow A$, implying $A \models e$.

2: Assume $e$ is equivalent to $\bigwedge E$, where $E \subseteq \operatorname{Sen}(\Sigma)$ such that for all $e^{\prime} \in E, e^{\prime}$ is $\mathcal{Q}$-preserved. Since $A_{k} \models e$, it holds that $A_{k} \models e^{\prime}$ for all $e^{\prime} \in E$, hence $A \models e^{\prime}$ for all $e^{\prime} \in E$, hence $A \models e$. The proof for disjunction goes similarly.

3: Assume $e$ is equivalent to $(\exists \varphi) e^{\prime}$, where $\varphi: \Sigma \rightarrow \Sigma^{\prime}$ is in $\mathcal{Q}$ and $e^{\prime}$ is $\mathcal{Q}$ preserved. Then there exists a $\varphi$-expansion $A_{k}^{\prime}$ of $A_{k}$ such that $A_{k}^{\prime} \models e^{\prime}$. By Lemma 4 , there exists a directed diagram $\left(A_{i}^{\prime} \stackrel{f_{i, j}^{\prime}}{\rightarrow} A_{j}^{\prime}\right)_{i, j \in I_{k}, i \leq j}$ in $\operatorname{Mod}\left(\Sigma^{\prime}\right)$, with colimit $\left(A_{i}^{\prime} \stackrel{\mu_{i}^{\prime}}{\rightarrow} A^{\prime}\right)_{i \in I_{k}}$ such that, for each $i, j \in I_{k}$ with $i \leq j, f_{i, j}^{\prime}$ is a $\varphi$-expansion of $f_{i, j}$ and $\mu_{i}^{\prime}$ is a $\varphi$-expansion of $\mu_{i}$. In particular, $A^{\prime} 1_{\varphi}=A$. According to Proposition 10.(3), each $f_{i, j}^{\prime}$ is $\mathcal{Q}$-elementary. Applying the fact that $e^{\prime}$ is $\mathcal{Q}$-preserved, we obtain that $A^{\prime}=e^{\prime}$, hence $A \models(\exists \varphi) e^{\prime}$, i.e., $A \models e$.

4: Assume $e$ is equivalent to $(\forall \varphi) e^{\prime}$, where $\varphi: \Sigma \rightarrow \Sigma^{\prime}$ is a finitely quasirepresentable signature morphism in $\mathcal{Q}$ and $e^{\prime}$ is $\mathcal{Q}$-preserved. Let $A^{\prime}$ be a $\varphi$ expansion of $A$. We need to show that $A^{\prime} \models e^{\prime}$. Because $\varphi$ is finitely quasirepresentable, there exists $q \in I$ and a $\varphi$-expansion $\xi^{\prime}: A_{q}^{\prime} \rightarrow A^{\prime}$ of $\mu_{q}$. Since $(I, \leq)$ is directed, there exists $p \in I$ such that $q \leq p$ and $k \leq p$. Thus, because $f_{k, p}$ is $\mathcal{Q}$-elementary, we get $A_{p} \models e$. Define $\left(A_{q}^{\prime} \stackrel{f^{\prime}}{\rightarrow} A_{p}^{\prime}, A_{p}^{\prime}\right)$ to be $\left(A_{q} / \operatorname{Mod}(\varphi)\right)^{-1}\left(A_{q} \stackrel{f_{q, p}}{\rightarrow} A_{p}, A_{p}\right)$ and $\nu^{\prime}$ to be $\left(A_{q} / \operatorname{Mod}(\varphi)\right)^{-1}\left(\mu_{p}:\left(A_{q}, f_{q, p}\right) \rightarrow\right.$ $\left.\left(A_{q}, \mu_{q}\right)\right)$. Note that $\nu^{\prime}: A_{p}^{\prime} \rightarrow A^{\prime}$. By Lemma 4 applied to the index $p$, there exists a directed diagram $\left(A_{i}^{\prime} \stackrel{f_{i, j}^{\prime}}{\rightarrow} A_{j}^{\prime}\right)_{i, j \in I_{p}, i \leq j}$ in $\operatorname{Mod}\left(\Sigma^{\prime}\right)$, with colimit $\left(A_{i}^{\prime} \stackrel{\mu_{i}^{\prime}}{\rightarrow} B^{\prime}\right)_{i \in I_{p}}$, such that, for each $i, j \in I_{p}$ with $i \leq j, f_{i, j}^{\prime}$ is a $\varphi$-expansion of $f_{i, j}$ and $\mu_{i}^{\prime}$ is a $\varphi$-expansion of $\mu_{i}$. Again, Proposition 10.(3) assures us that each $f_{i, j}^{\prime}$ is $\mathcal{Q}$-elementary. Since both $\left(\nu^{\prime}, A^{\prime}\right)$ and $\left(\mu_{p}^{\prime}, B^{\prime}\right)$ are equal to $\left(A_{p} / \operatorname{Mod}(\varphi)\right)^{-1}\left(\mu_{p}, A\right)$, it follows that $A^{\prime}=B^{\prime}$ and $\nu^{\prime}=\mu_{p}^{\prime}$. Finally, since $e^{\prime}$ is $\mathcal{Q}$-preserved and $A_{p}^{\prime} \models e^{\prime}\left(A_{p}^{\prime}\right.$ being a $\varphi$-expansion of $\left.A_{p}\right)$, we obtain that $A^{\prime} \models e^{\prime}$.

q.e.d.

Proposition 12 Assume that the institution admits negation. Then the class of sentences preserved and reflected by directed colimits of $\mathcal{Q}$-elementary morphisms 
1. contains all finitary basic sentences,

2. is closed under arbitrary conjunction and disjunction, and under negation,

3. is closed under universal and existential quantification over finitely quasirepresentable morphisms in $\mathcal{Q}$.

Proof:

Let $e \in \operatorname{Sen}(\Sigma),\left(A_{i} \stackrel{f_{i, j}}{\rightarrow} A_{j}\right)_{i, j \in I, i \leq j}$ a directed diagram of $\mathcal{Q}$-elementary $\Sigma$ morphisms, with colimit $\left(A_{i} \stackrel{\mu_{i}}{\longrightarrow} A\right)_{i \in I}$, and let $k \in I$. We need to prove $\left[A_{k} \models e\right.$ iff $A=e]$.

1: Assume $e$ is a finitary basic sentence. That $A_{k} \models e$ implies $A \models e$ follows from Proposition 11.(1). Therefore let us suppose $A \models e$, i.e., there exists a $\Sigma$-morphism $g: M_{e} \rightarrow A$, and let us show that $A_{k}=e$. Because $M_{e}$ is finitely presentable, there exists $j \in I$ and $\Sigma$-morphism $h: M_{e} \rightarrow A_{j}$ such that $h ; \mu_{j}=g$. Because $(I, \leq)$ is directed, there exists $i \in I$ such that $k \leq i$ and $j \leq i$. Then, by the existence of the morphism $M_{e} \stackrel{h ; f_{j, i}}{\rightarrow} A_{i}$, it follows that $A_{i}=e$. Moreover, since $f_{k, i}: A_{k} \rightarrow A_{i}$ is $\mathcal{Q}$-elementary and the institution admits negation, we obtain $A_{k} \models e$.

2: Similar to the proof of Proposition 11.(2) for conjunction and disjunction. For negation, the property is obvious thanks to its symmetry.

3: Because the institution admits negation, universal and existential quantifications are mutually definable. Therefore let us focus on existential quantification. Assume $e$ is equivalent to $(\forall \varphi) e^{\prime}$, where $\varphi: \Sigma \rightarrow \Sigma^{\prime}$ is a finitely quasirepresentable morphism in $\mathcal{Q}$ and $e^{\prime}$ is a $\Sigma^{\prime}$-sentence $\mathcal{Q}$-[preserved and reflected]. That $A_{k}=e$ implies $A=e$ follows from Proposition 11.(3). Let us now suppose $A \models(\exists \varphi) e^{\prime}$ and let us show that $A_{k} \models(\exists \varphi) e^{\prime}$. We have that $A \models \neg(\forall \varphi) \neg e^{\prime}$, which means $A \not \models(\forall \varphi) \neg e^{\prime}$. By point (2), $\neg e^{\prime}$ is $\mathcal{Q}$-[preserved and reflected]. Thus, by Proposition 11.(4), $(\forall \varphi) \neg e^{\prime}$ is $\mathcal{Q}$-preserved, hence $A_{k} \models(\forall \varphi) \neg e^{\prime}$ would imply $A \models(\forall \varphi) \neg e^{\prime}$, which is a contradiction. Thus $A_{k} \models \neg(\forall \varphi) \neg e^{\prime}$, i.e., $A_{k} \models(\exists \varphi) e^{\prime}$. q.e.d.

Proposition 13 Assume that all sentences of the institution are preserved by directed colimits of $\mathcal{Q}$-elementary morphisms. Then for each signature $\Sigma$, the subcategory of $\operatorname{Mod}(\Sigma)$ of $\mathcal{Q}$-elementary morphisms is closed under directed colimits.

Proof:

Let $\left(A_{i} \stackrel{f_{i, j}}{\rightarrow} A_{j}\right)_{i, j \in I, i \leq j}$ be a directed diagram in $\operatorname{Mod}(\Sigma)$ such that each $f_{i, j}$ is $\mathcal{Q}$-elementary and let $\left(A_{i} \stackrel{\mu_{i}}{\rightarrow} A\right)_{i \in I}$ be its colimit. Let $k \in I$. In order to prove that $\mu_{k}$ is $\mathcal{Q}$-elementary, let $\nu^{\prime}: A_{k}^{\prime} \rightarrow A^{\prime}$ be a $\varphi$-expansion of $\mu_{k}$ and let $e^{\prime} \in \operatorname{Sen}\left(\Sigma^{\prime}\right)$ such that $A_{k}^{\prime}=e^{\prime}$. By Lemma 4 , there exists a directed diagram $\left(A_{i}^{\prime} \stackrel{f_{i, j}^{\prime}}{\rightarrow} A_{j}^{\prime}\right)_{i, j \in I_{k}, i \leq j}$ in $\operatorname{Mod}\left(\Sigma^{\prime}\right)$, with colimit $\left(A_{i}^{\prime} \stackrel{\mu_{i}^{\prime}}{\rightarrow} B^{\prime}\right)_{i \in I_{k}}$, such that, for each $i, j \in I_{k}$ with $i \leq j, f_{i, j}^{\prime}$ is a $\varphi$-expansion of $f_{i, j}$ and $\mu_{i}^{\prime}$ is a $\varphi$-expansion of $\mu_{i}$. Just like in the proof of Proposition 11.(4), one obtains that each $f_{i, j}^{\prime}$ is $\mathcal{Q}$-elementary and that $A^{\prime}=B^{\prime}$ and $\nu^{\prime}=\mu_{k}^{\prime}$. Thus, according to our hypothesis, $A^{\prime} \models e^{\prime}$. 
q.e.d.

Definition 14 An institution $\mathcal{I}$ is called $\mathcal{Q}$-first-order-accessible if one of the two below properties holds:

1. All sentences of $\mathcal{I}$ are (equivalent to ones) obtained from basic sentences by applying a finite number of times the following rules:

- arbitrary conjunction;

- arbitrary disjunction;

- existential quantification over morphisms in $\mathcal{Q}$;

- universal quantification over finitely quasi-representable morphisms in $\mathcal{Q}$.

2. $\mathcal{I}$ admits negation and all sentences of $\mathcal{I}$ are (equivalent to ones) obtained from finitary basic sentences by applying a finite number of times the following rules:

- arbitrary conjunction;

- arbitrary disjunction;

- negation;

- existential quantification over finitely quasi-representable morphisms in $\mathcal{Q}$

- universal quantification over finitely quasi-representable morphisms in $\mathcal{Q}$.

Proposition 15 All the examples 1-14 of institutions are $\mathcal{Q}$-first-order-accessible, where $\mathcal{Q}$ is each time the category of injective signature morphisms that only add constants.

Proof:

Let us first see that IFOPL and all its fragments are $\mathcal{Q}$-first-order accessible. Indeed, for each signature $\Sigma=(S, F, P)$, an equality atom $t=t^{\prime}$ is finitary basic thanks to the model $T_{\Sigma} / t=t^{\prime}$, that is, the $(S, F)$-algebra $T_{F} / t=t^{\prime}$ (a quotient of the ground term algebra over $F$ ) with all the relations in $P$ empty, while a relational atom $R\left(t_{1}, \ldots, t_{n}\right)$ is basic thanks to the model consisting of $T_{F}$ together with all relations in $P$ empty, except $R$, which is the singleton $\left\{\left(t_{1}, \ldots, t_{n}\right)\right\}$. Moreover, quantification over finite or infinite sets of variables are particular cases of quantification over signature morphisms in $\mathcal{Q}$. Thus PosFOPL, EQL, and $M L$ fall into case 1 of Definition 14, and FOPL, $(\Pi \cup \Sigma)_{n}^{0}, I F O P L, I F O P L_{\alpha}$, and $E Q L N$ into case 2.

A similar argument as above holds for $P F O P L$ and $P A$ too, since for instance given a $P A$-signature and a set of equality atoms, there exists the initial algebra in the category of algebras satisfying these atoms [3]. And similarly for $R W L$ and $O S L$.

As for $E H L$ and $H L$, one has to notice mainly two things. First, all usual equality atoms are basic; indeed, the algebra $T_{F} / t=t^{\prime}$ has the property that, for each $(H, V, F)$-model $A$ satisfying the usual equality atom $t=t^{\prime}$, the unique $E Q L$-morphism between $T_{F} / t=t^{\prime}$ and $A$ preserves behavioral equivalence, hence it is also an $E H L$ - and $H L$ - morphism. Second, the behavioral equality atoms are equivalent to (infinite) conjunctions of universally quantified usual equality atoms; indeed, it holds that $A=t \equiv t^{\prime}$ iff $A=\Lambda\left\{\left(\forall\left\{x_{1}, \ldots, x_{n}\right\}\right) c[z \leftarrow\right.$ $t]=c\left[z \leftarrow t^{\prime}\right] \mid v \in V, x_{1}, \ldots, x_{n}$ variables, $c \in T_{F}\left(\left\{x_{1}, \ldots, x_{n}, z\right\}\right)_{v}$ context $\}$. 
Thus $H L$ falls into case 1 of Definition 14. Moreover, $E H L$ falls into case 2, since although behavioral equality atoms are not finitary, they are nevertheless obtainable from finitary basic sentences by means of the rules of universal quantification over finitely quasi-representable morphisms in $\mathcal{Q}$ (i.e., over finite sets of variables) and arbitrary conjunction.

q.e.d.

Theorem 16. (Elementary Chain Theorem) Assume that the institution is $\mathcal{Q}$ first-order-accessible. Then for each signature $\Sigma$, the subcategory of $\operatorname{Mod}(\Sigma)$ of $\mathcal{Q}$-elementary morphisms is closed under directed colimits. In particular, the institution enjoys the $\mathcal{Q}-E C P$.

Proof:

Follows immediately: for case 1 of Definition 14 from Propositions 11 and 13, and for case 2 from Propositions 12 and 13.

q.e.d.

The separation on two cases in Definition 14 covers mainly the following situations: the institution $\mathcal{I}$ either admits negation, or has no negation - intermediate cases are not covered. Some important examples of institutions to which our Theorem 16 does not apply are all variations of Horn logic - in fact, for those institutions, we conjecture that the elementary chain property does not hold.

Corollary 17 All the examples 1-14 of institutions enjoy the $\mathcal{Q}-E C P$.

Note that Theorem 16 is applicable to a whole variety of other logics resulted from other different combinations of connectives and quantifiers. An interesting example which takes full advantage of Proposition 11 is a version of positive infinitary first-order logic admitting arbitrary conjunction and disjunction, existential quantification over arbitrary sets of variables, and universal quantification over finite sets of variables. Moreover, the case of fragments of languages (over transitive sets) in infinitary first-order logic [18] also seems to fall into our framework, provided that one takes the trouble of formalizing this as an institution.

\section{Elementary morphisms by diagrams}

An alternative definition of elementary embeddings in classical model theory is given in terms of elementary diagrams $[4,25]$. There, the elementary diagram $E D g(A)$ of a model $A$ is the set of all sentences in $\Sigma(A)$ (the language $\Sigma$ of $A$ extended with all elements of $A$ as constants), true in $A$. Then an embedding $A \stackrel{h}{\rightarrow} B$ is elementary iff $h(E D g(A)) \subseteq E D g(B)$, where $h(E D g(A))$ is the obvious translation through $h$ of the sentences in $E D g(A)$. The main difference to the original definition (discussed at the beginning of Section 4 ) is that a language which includes parameter symbols for the source model, $\Sigma(A)$, is a priori given and the desired property is stated locally, in that fixed language. By adapting an existing institutional concept of diagram, we can discuss this alternative definition in a logic-independent framework. 


\section{Institutional Diagrams}

Diagrams are a basic concept in classical model theory [4]. They were first generalized to the institutional framework in $[35,36]$; there, it is defined the concept of abstract algebraic institution, which is an institution subject to some additional natural requirements (like finite-exactness, existence of direct products of models etc.) and enriched with a system of diagrams. The reason for introducing diagrams there was making all algebras accessible, for specification purposes. In this paper, we need a more elaborated notion of institutional diagram, defined in [8], which takes into consideration not only models, but also model morphisms. The concept was introduced under the name "elementary diagram". For reasons that will be pointed out soon, here we prefer to use, like in [35], the name "positive diagram" instead.

An institution $\mathcal{I}=(\operatorname{Sign}, \operatorname{Sen}, \operatorname{Mod}, \models)$ is said to have positive diagrams [8] if

1. for each signature $\Sigma$ and $\Sigma$-model $A$ there exists a signature morphism $\iota_{\Sigma}(A): \Sigma \rightarrow \Sigma_{A}$ and a set $E_{A}$ of $\Sigma_{A}$-sentences (called the positive diagram of $A$ ) such that $\operatorname{Mod}\left(\Sigma_{A}, E_{A}\right)$ and $A / \operatorname{Mod}(\Sigma)$ are isomorphic by an isomorphism $i_{\Sigma, A}$ making the following diagram commutative:

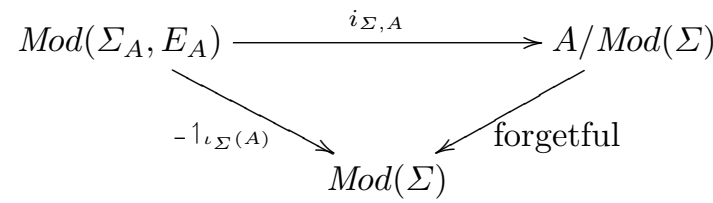

2. $\iota$ is "functorial", i.e., for each signature morphism $\varphi: \Sigma \rightarrow \Sigma^{\prime}$, each $A \in$ $|\operatorname{Mod}(\Sigma)|, A^{\prime} \in\left|\operatorname{Mod}\left(\Sigma^{\prime}\right)\right|$ and $h: A \rightarrow A^{\prime} 1_{\varphi}$ in $\operatorname{Mod}(\Sigma)$, there exists a presentation morphism $\iota_{\varphi}(h):\left(\Sigma_{A}, E_{A}\right) \rightarrow\left(\Sigma_{A^{\prime}}^{\prime}, E_{A^{\prime}}\right)$ making the following diagram commutative:

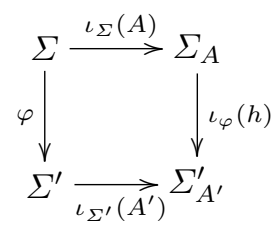

3. $i$ is natural, i.e., for each signature morphism $\varphi: \Sigma \rightarrow \Sigma^{\prime}$, each $A \in$ $|\operatorname{Mod}(\Sigma)|, A^{\prime} \in\left|\operatorname{Mod}\left(\Sigma^{\prime}\right)\right|$ and $h: A \rightarrow A^{\prime} 1_{\varphi}$ in $\operatorname{Mod}(\Sigma)$, the following diagram is commutative:




Here are some notational conventions that we hope will make the reader's life easier. Let $\varphi: \Sigma \rightarrow \Sigma^{\prime}$ be a signature morphism, $A^{\prime} \in\left|\operatorname{Mod}\left(\Sigma^{\prime}\right)\right|$, and $h: A \rightarrow B$ in $\operatorname{Mod}(\Sigma)$. We write $\iota_{\Sigma}(h)$ instead of $\iota_{1_{\Sigma}}(h)$ and $\iota_{\varphi}\left(A^{\prime} 1_{\varphi}\right)$ instead of $\iota_{\varphi}\left(1_{\left(A^{\prime} 1_{\varphi}\right)}\right)$. Let $A$ be a fixed object in $\operatorname{Mod}(\Sigma)$ and let $B, C \in|\operatorname{Mod}(\Sigma)|$ and $f: A \rightarrow B$, $g: A \rightarrow C, u: B \rightarrow C$ morphisms in $\operatorname{Mod}(\Sigma)$ such that $f ; u=g$. Then $(f, B)$ and $(g, C)$ are objects in $A / \operatorname{Mod}(\Sigma)$ and $u$ is also a morphism in $A / \operatorname{Mod}(\Sigma)$ between $(f, B)$ and $(g, C)$. We establish the following notations: $B_{f}=i_{\Sigma, A}^{-1}(f, B)$ (and, similarly, $\left.C_{g}=i_{\Sigma, A}^{-1}(g, C)\right), u_{f, g}=i_{\Sigma, A}^{-1}((f, B) \stackrel{u}{\rightarrow}(g, C))$. Thus, for instance, let $f: A \rightarrow B$ be a $\Sigma$-model morphism. Then $f_{1_{A}, f}$ is the image through $i_{\Sigma, A}^{-1}$ of the morphism $f:\left(1_{A}, A\right) \rightarrow(f, B)$ in $A / \operatorname{Mod}(\Sigma)$, and has source $A_{\left(1_{A}\right)}$ and target $B_{f}$. We shall write $A_{A}$ instead of $A_{\left(1_{A}\right)}$ and $f_{A, f}$ instead of $f_{1_{A}, f}$.

In classical model theory, $\Sigma_{A}$ is the signature $\Sigma$ enriched with all the elements of $A$ as constants, $\iota_{\Sigma}(A): \Sigma \rightarrow \Sigma_{A}$ is the inclusion of signatures, and $E_{A}$ is a set of parameterized sentences which hold in $A$, depending on the considered type of morphism between models. If arbitrary model-homomorphisms are allowed as morphisms, like in FOPL, one gets the "positive diagram"; if just modelembeddings are considered, one gets the "diagram"; and only if just elementary embeddings are allowed, one gets what is classically called "elementary diagram" (see [4] for the pointed standard terminology in classical model theory). Thus the above institutional definition of diagrams particularizes to elementary diagrams for classical first-order logic only if a notion of elementary morphism is assumed as previously defined. However, it is precisely the latter notion that we want to capture using diagrams. Therefore, we prefer to use the term "positive diagram", in accordance to the particularization of the concept to the concrete institution FOPL, widely accepted as the institution of first-order logic. Thus we view the set of sentences $E_{A}$ as the positive, rather than elementary, diagram of $A$, but of course keeping for it the same understanding as in [8]: that $E_{A}$ axiomatizes the class of $\Sigma$-morphisms with source $A$. And we use the term elementary diagram of $A$ for the set $\left\{A_{A}\right\}^{*}$, of all sentences satisfied by the self-parameterized extension $A_{A}$ of $A$.

In [8], there are presented positive diagrams for FOPL, RWL, PA, and $H L$. Most institutions that were built starting from "working" logical systems tend to have elementary diagrams. We next recall the system of positive diagrams for $F O P L$. Let $\Sigma=(S, F, P)$ be a $F O P L$-signature and $A \in|\operatorname{Mod}(\Sigma)|$. Define $\Sigma_{A}=\left(S, F_{A}, P\right)$, where $F_{A}$ extends $F$ by adding, for each $s \in S$, all elements of $A_{s}$ as constants of sort $s$. Further, we define:

- $A_{A} \in\left|\operatorname{Mod}\left(\Sigma_{A}\right)\right|$, as the $\Sigma_{A^{-}}$expansion of $A$ which interprets each constant $a \in A$ by $a$;

- $E_{A}$, as the set of all atoms in $\operatorname{Sen}\left(\Sigma_{A}\right)$ satisfied by $A_{A}$;

$-\iota_{\Sigma}(A)$, as the signature inclusion of $\Sigma$ into $\Sigma_{A}$;

- the functor $i_{\Sigma, A}: \operatorname{Mod}\left(\Sigma_{A}, E_{A}\right) \rightarrow A / \operatorname{Mod}(\Sigma)$, as:

- $i_{\Sigma, A}\left(B^{\prime}\right)=(A \stackrel{h}{\rightarrow} B, B)$, where $B=B^{\prime} 1_{\iota_{\Sigma}(A)}$ and, for each $s \in S$ and $a \in A_{s}, h_{s}(a)=B_{a}^{\prime}$.

- $i_{\Sigma, A}(f)=f$. 
Let $\varphi: \Sigma=(S, F, P) \rightarrow \Sigma^{\prime}=\left(S^{\prime}, F^{\prime}, P^{\prime}\right)$ be a signature morphism, $A \in$ $|\operatorname{Mod}(\Sigma)|, B \in\left|\operatorname{Mod}\left(\Sigma^{\prime}\right)\right|$, and $h: A \rightarrow B 1_{\varphi}$ in $\operatorname{Mod}(\Sigma)$. Then the natural presentation morphism $\iota_{\varphi}(h):\left(\Sigma_{A}, E_{A}\right) \rightarrow\left(\Sigma_{B}^{\prime}, E_{B}\right)$ from the definition of positive diagrams is the following: if $e \in \operatorname{Sen}\left(\Sigma_{A}\right)$, then $\iota_{\varphi}(h)(e)$ is obtained from $e$ by symbol-wise translation, mapping:

- each $\sigma \in F$ into $\varphi^{o p}(\sigma)$,

- each $R \in P$ into $\varphi^{r e l}(R)$,

- for all $s \in S$, each $a \in A_{s}$ into $h_{s}(a)$,

- for all $s \in S$, each variable $x: s$ of sort $s$ into a variable $x: \varphi^{\text {sort }}(s)$ of sort $\varphi^{\text {sort }}(s)$

- each other symbol $u$ that appears in $e$ (e.g., logical connectives and quantifiers) into $u$.

As a general rule easily seen to hold about positive diagrams, one has that:

- if an institution $\mathcal{I}^{\prime}$ extends an institution $\mathcal{I}$ and has the same category Sign and functor $\operatorname{Mod}$ (thus only adds new sentences), then positive diagrams are inherited by $\mathcal{I}^{\prime}$ from $\mathcal{I}$;

- if an institution $\mathcal{I}^{\prime}$ restricts an institution $\mathcal{I}$, has the same category Sign and functor $\operatorname{Mod}$ (thus only restricts the sets of sentences), but $\mathcal{I}^{\prime}$ still has all the sentences in the positive diagrams $E_{A}$ of $\mathcal{I}$, then positive diagrams are inherited by $\mathcal{I}^{\prime}$ from $\mathcal{I}$.

Thus the above described positive diagrams of FOPL are inherited by $I F O P L$, $I F O L P L_{\alpha}$, PosFOPL, EQL, EQLN, ML. The positive diagrams for the other mentioned institutions can be constructed with a similar pattern as those of FOPL; as remarked in [8], the sentences $E_{A}$ are always the basic sentences satisfied by the model $A$ expanded to $\Sigma_{A}$ with constants in $A$ pointing to themselves.

For what follows, we fix an institution with positive diagrams, $\mathcal{I}$.

Definition 18 Let $\Sigma$ be a signature.

1. Given a $\Sigma$-model $A$, the elementary diagram of $A$ is the set $\left\{A_{A}\right\}^{*}$ (of $\Sigma_{A^{-}}$sentences satisfied by $\left.A_{A}\right){ }^{6}$

2. A $\Sigma$-morphism $A \stackrel{h}{\rightarrow} B$ is called elementary by diagrams (d-elementary) if one of the following two equivalent conditions holds:

$-A_{A}^{*} \subseteq B_{h}^{*}$

$-\iota_{\Sigma}(h)\left(A_{A}^{*}\right) \subseteq B_{B}^{*}$.

That the two conditions in point (2) of the above definition are equivalent follows from the satisfaction condition together with the fact that, by the naturality of $i, B_{B} 1_{\iota_{\Sigma}(h)}=B_{h}$.

Thus we defined elementary morphisms by means of elementary diagrams. We can spell out this definition as follows: $A \stackrel{h}{\rightarrow} B$ is d-elementary if the elementary diagram of $A$ is embedded, via $h$, into the elementary diagram of $B$.

\footnotetext{
${ }^{6}$ Since $A_{A} \models E_{A}$, the positive diagram of $A$ is included in the elementary diagram of $A$.
} 


\section{The relationship between $\mathcal{Q}$-elementary and d-elementary}

The notion of d-elementary morphism is more compact than that of $\mathcal{Q}$-elementary morphism, but the former needs a lot of further structure on top of the plain institutional structure. We next provide conditions under which the two concepts are equivalent. For all this section, we fix a broad subcategory $\mathcal{Q}$ of Sign consisting of representable signature morphisms. ${ }^{7}$

Definition 19 The positive diagrams of $\mathcal{I}$ are said to be $\mathcal{Q}$-normal if for each representable signature morphism $\varphi: \Sigma \rightarrow \Sigma^{\prime}$ (represented by $M_{\varphi}$ ) there exists a signature morphism $\chi: \Sigma^{\prime} \rightarrow \Sigma_{M_{\varphi}}$ such that $\varphi ; \chi=\iota_{\Sigma}\left(M_{\varphi}\right)$ and $\operatorname{Mod}(\chi) ; I_{\varphi}=$ $i_{\Sigma, M_{\varphi}} \cdot{ }^{8}$

In examples 1-14 of institutions, for the usual choice of $\mathcal{Q}$, i.e., to consist of all injective signature morphisms that only add constants, the signature morphisms $\iota_{\Sigma}(A)$ of the positive diagrams are all in $\mathcal{Q}$. Moreover, in each case, the positive diagrams are also $\mathcal{Q}$-normal. Indeed, for example in FOPL, given an injective signature morphism $\varphi$ that only adds constants, which we can assume w.l.g. to be an inclusion $\Sigma=(S, F, P) \rightarrow \Sigma^{\prime}=(S, F \cup X, P)$, represented by the $\Sigma$-model $T_{\Sigma}(X)$, the desired morphism $\chi$ such that $\varphi ; \chi=\iota_{\Sigma}\left(T_{\Sigma}(X)\right)$ is the signature inclusion $(S, F \cup X, P) \rightarrow\left(S, F \cup T_{\Sigma}(X), P\right)$ given by the set inclusion $X \rightarrow$ $T_{\Sigma}(X)$. In order to see that the corresponding condition on model categories holds, let $N$ be a $\Sigma_{T_{\Sigma}(X)}$-model that satisfies $E_{T_{\Sigma}(X)}$. Then $i_{\Sigma, T_{\Sigma}(X)}(N)=(h$ : $\left.T_{\Sigma}(X) \rightarrow N 1_{\iota_{\Sigma}\left(T_{\Sigma}(X)\right)}, N 1_{\iota_{\Sigma}\left(T_{\Sigma}(X)\right)}\right)$, where $h(t)=N_{t}$ for all $t \in T_{\Sigma}(X)$. On the other hand, $I_{\varphi}\left(N 1_{\chi}\right)=\left(g: T_{\Sigma}(X) \rightarrow N 1_{\varphi ; \chi}, N 1_{\varphi ; \chi}\right)=\left(g: T_{\Sigma}(X) \rightarrow\right.$ $\left.N 1_{\iota_{\Sigma}\left(T_{\Sigma}(X)\right)}, N 1_{\iota_{\Sigma}\left(T_{\Sigma}(X)\right)}\right)$, where $g$ is the unique $\Sigma$-morphism extending the mapping $v: X \rightarrow N$ defined by $v(x)=N_{x}$ for all $x \in X$. Thus, by the freeness of $T_{\Sigma}(X), g=h$. Hence the functors $\operatorname{Mod}(\chi) ; I_{\varphi}$ and $i_{\Sigma, M_{\varphi}}$ coincide on objects. That they coincide on morphisms too follows at once from $\varphi ; \chi=\iota_{\Sigma}\left(T_{\Sigma}(X)\right)$. Normality of the positive diagrams for the other examples of institutions can be shown similarly to the case of FOPL.

Proposition 20 If the positive diagrams are normal and have each signature morphism $\iota_{\Sigma}(A)$ in $\mathcal{Q}$, then any model morphism is $\mathcal{Q}$-elementary iff it is $d$ elementary.

\section{Proof:}

Let $\Sigma$ be a signature and $A \stackrel{h}{\rightarrow} B$ a $\Sigma$-morphism.

Assume first that $h$ is $\mathcal{Q}$-elementary. Then, since $\iota_{\Sigma}(A)$ is in $\mathcal{Q}$ and $A_{A} \stackrel{h_{A}}{\rightarrow} B_{h}$ is a $\iota_{\Sigma}(A)$-expansion of $h$, we get $\left\{A_{A}\right\}^{*} \subseteq\left\{B_{h}\right\}^{*}$. Thus $h$ is d-elementary.

Conversely, assume that $h$ is d-elementary. Let $\varphi: \Sigma \rightarrow \Sigma^{\prime}$ be a signature morphism in $\mathcal{Q}$ and $A^{\prime} \stackrel{h^{\prime}}{\rightarrow} B^{\prime}$ a $\varphi$-expansion of $h$. Let $\left(M_{\varphi} \stackrel{a}{\rightarrow} A, A\right)=I_{\varphi}\left(A^{\prime}\right)$ and

\footnotetext{
${ }^{7}$ Note that we require more than usual for the subcategory $\mathcal{Q}$, namely representability instead of quasi-representability.

${ }^{8}$ Here, we made the slight notational abuse of letting $\operatorname{Mod}(\chi)$ denote the restriction of $\operatorname{Mod}(\chi): \operatorname{Mod}\left(\Sigma_{M_{\varphi}}\right) \rightarrow \operatorname{Mod}\left(\Sigma^{\prime}\right)$ to $\operatorname{Mod}\left(\Sigma_{M_{\varphi}}, E_{M \varphi}\right)$.
} 
$\left(M_{\varphi} \stackrel{b}{\rightarrow} B, B\right)=I_{\varphi}\left(B^{\prime}\right)$. By the naturality of $i$ we have that $h_{A, h} 1_{\iota_{\Sigma}(a)}=h_{a, b} \cdot{ }^{9}$ Since $\left\{A_{A}\right\}^{*} \subseteq\left\{B_{h}\right\}^{*}$, by the satisfaction condition, it follows that $\left\{A_{a}\right\}^{*} \subseteq$ $\left\{B_{b}\right\}^{*}$. Now, by the normality of diagrams, there exists $\chi: \Sigma^{\prime} \rightarrow \Sigma_{M_{\varphi}}$ such that $\varphi ; \chi=\iota_{\Sigma}\left(M_{\varphi}\right)$ and $\operatorname{Mod}(\chi) ; I_{\varphi}=i_{\Sigma, M \varphi}$. Then $\operatorname{Mod}(\chi)=i_{\Sigma, M \varphi} ; I_{\varphi}^{-1}$, thus $h_{a, b} 1_{\chi}=I_{\varphi}^{-1}\left(i_{\Sigma, M_{\varphi}}\left(h_{a, b}\right)\right)=I_{\varphi}^{-1}(h)=h^{\prime}$. Hence $A_{a} 1_{\chi}=A^{\prime}$ and $B_{b} 1_{\chi}=B^{\prime}$. Finally, by the satisfaction condition, we get $\left\{A^{\prime}\right\}^{*} \subseteq\left\{B^{\prime}\right\}^{*}$.

q.e.d.

Corollary 21 In all the examples 1-14 of institutions (with their mentioned diagrams), a model morphism is elementary iff it is d-elementary.

\section{Concluding remarks}

We outline the contributions of the present paper:

- introduced an abstract notion of elementary morphism, parameterized by a class of signature morphisms;

- studied the connection between elementary morphisms and positive diagrams in an arbitrary institution, by giving an alternative diagrammatic definition of elementarity;

- showed how the general results particularize to many concrete cases of logical systems, yielding different known results in a unitary fashion, but also some new results; in particular, the less conventional cases of partial algebra, hidden logic and rewriting logic fall into our framework.

An open problem that we consider worthwhile is the institutional relationship between elementary morphisms and model-embeddings. Given the fact that classically elementary morphisms are also embeddings, a result stating that, under certain assumptions on the expressive power of sentences, all elementary morphisms are embeddings (where "embeddings" can be defined either strictly categorically, as subobjects, or by means of inclusion or factorization systems), would be very desirable.

A more in depth study of elementary morphisms in some particular cases might also prove to be interesting. Take for instance the equational framework. In $E Q L$ and $E Q L N$, the elementary morphisms do not seem very amenable. It is not clear to us how they look like. Note that a surjective morphism is always elementary in $E Q L$, and an elementary morphism has to be injective in $E Q L N$. The case of $H L$ is even more intricate, and the notion of "elementary behavioral morphism", complementing that of bisimulation, is potentially fruitful in the algebraic study of systems and behavior.

\footnotetext{
${ }^{9} h_{A, h}: A_{A} \rightarrow B_{h}$ is a morphism in $\Sigma_{A}$ and $h_{a, b}: A_{a} \rightarrow B_{b}$ is a morphism in $\Sigma_{M_{\varphi}}$ -
} recall the notational conventions about elementary diagrams. 


\section{Acknowledgments}

Răzvan Diaconescu taught us institution-independent model theory, during some lectures that he held at Şcoala Normală Superioară, Romania. He also read several preliminary versions of this paper, and his comments improved the paper substantially. Our colleagues, especially Traian Şerbănuţă, Marius Petria, and Cristian Cucu, pointed out lots of bugs in our proofs and minds w.r.t. elementary chains, that we hope we managed to correct. Last but not least, our two referees criticized the paper constructively.

\section{References}

1. J. Barwise and J. Feferman. Model-Theoretic Logics. Springer, 1985.

2. M. Bidoit and R. Hennicker. Proving behavioural theorems with standard firstorder logic. In Algebraic and Logic Programming (ALP'94), volume 850 of LNCS, pages 41-58, 1994.

3. P. Burmeister. A Model Theoretic Oriented Appraoch to Partial Algebras. Akademie-Verlag Berlin, 1986.

4. C. C. Chang and H. J. Keisler. Model Theory. North Holland, Amsterdam, 1973.

5. M. Clavel, S. Eker, P. Lincoln, and J. Meseguer. Principles of Maude. In J. Meseguer, editor, Proceedings of the First International Workshop on Rewriting Logic, volume 4 of Electronic Notes in Theoretical Computer Science, pages 65-89. Elsevier, 1996.

6. R. Diaconescu. Institution-independent Model Theory. Book draft. (Ask author for current version at Razvan.Diaconescu@imar.ro).

7. R. Diaconescu. Institution-independent ultraproducts. Fundamenta Informaticae, $55(3-4): 321-348,2003$.

8. R. Diaconescu. Elementary diagrams in institutions. Journal of Logic and Computation, 14(5):651-674, 2004.

9. R. Diaconescu. Herbrand theorems in arbitrary institutions. Inf. Process. Lett., 90:29-37, 2004.

10. R. Diaconescu. An institution-independent proof of Craig interpolation theorem. Studia Logica, 77(1):59-79, 2004.

11. R. Diaconescu and K. Futatsugi. CafeOBJ Report: The Language, Proof Techniques, and Methodologies for Object-Oriented Algebraic Specification. World Scientific, 1998. AMAST Series in Computing, volume 6.

12. R. Diaconescu and K. Futatsugi. Behavioural coherence in object-oriented algebraic specification. Universal Computer Science, 6:74-96, 2000.

13. D. Găină and A. Popescu. An institution-independent proof of Robinson consistency theorem. Studia Logica. To appear.

14. J. Goguen and R. Burstall. Institutions: Abstract model theory for specification and programming. Journal of the Association for Computing Machinery, 39(1):95146, January 1992.

15. J. Goguen and G. Malcolm. A hidden agenda. Theoretical Computer Science, 245(1):55-101, August 2000.

16. J. Goguen and G. Roşu. Hiding more of hidden algebra. In Formal Methods 1999 (FM'99), volume 1709 of Lecture Notes in Computer Sciences, pages 1704-1719. Springer-Verlag, 1999. 
17. J. Hirschfeld and W. Wheeler. Forcing, arithmetic, division rings. Lecture Notes in Mathematics, (454), 1975.

18. H. J. Keisler. Model Theory for Infinitary Logic. North-Holland, 1971.

19. S. Mac Lane. Categories for the Working Mathematician. Springer, 1971.

20. M. Makkai and R. Paré. Accessible Categories: The Foundations of Categorical Model Theory. Providence, 1989.

21. M. Makkai and G. Reyes. First order categorical logic. Lecture Notes in Mathematics, (611), 1977.

22. J. Meseguer. General logics. In H.-D. Ebbinghaus et al., editors, Proceedings, Logic Colloquium 1987, pages 275-329. North-Holland, 1989.

23. J. Meseguer. Conditional Rewriting Logic as a Unified Model of Concurrency. Theoretical Computer Science, pages 73-155, 1992.

24. J. Meseguer. Membership algebra as a logical framework for equational specification. In Proceedings, WADT'97, volume 1376 of LNCS, pages 18-61. Springer, 1998.

25. J. D. Monk. Mathematical Logic. Springer-Verlag, 1976.

26. T. Mossakowski, J. Goguen, R. Diaconescu, and A. Tarlecki. What is a logic? In J.-Y. Beziau, editor, Logica Universalis, pages 113-133. Birkhauser, 2005.

27. M. Petria and R. Diaconescu. Abstract Beth definability institutionally. Submitted. (Ask authors for current version at Razvan.Diaconescu@imar.ro).

28. H. Reichel. Structural Induction on Partial Algebras. Akademie-Verlag Berlin, 1984.

29. G. Roşu. The institution of order-sorted equational logic. Bulletin of EATCS, 53:250-255, 1994.

30. G. E. Sacks. Saturated Model Theory. W. A. Bejamin, Inc., Reading, 1972.

31. A. Salibra and G. Scollo. A soft stairway to institutions. In M. Bidoit and C. Choppy, editors, Recent Trends in Data Type Specification, volume 655 of Lecture Notes in Computer Science, pages 310-329. Springer-Verlag, 1992.

32. A. Salibra and G. Scollo. Interpolation and compactness in categories of preinstitutions. Math. Struct. in Comp. Science, 6:261-286, 1996.

33. T. Şerbănuţă. Institutional concepts in first-order logic, parameterized specifications, and logic programming. Master Disertation. University of Bucharest, 2004.

34. A. Tarlecki. Bits and pieces of the theory of institutions. In D. Pitt, S. Abramsky, A. Poigné, and D. Rydeheard, editors, Proceedings, Summer Workshop on Category Theory and Computer Programming, Lecture Notes in Computer Science, volume 240, pages 334-360. Springer, 1986.

35. A. Tarlecki. On the existence of free models in abstract algebraic institutions. Theoretical Computer Science, 37:269-304, 1986.

36. A. Tarlecki. Quasi-varieties in abstract algebraic institutions. Journal of Computer and System Sciences, 33(3):333-360, 1986. 